\title{
Ophiostomatoid fungi associated with mites phoretic on bark beetles in Qinghai, China
}

Runlei Chang ${ }^{1,2}$, Tuan A. Duong ${ }^{1}$, Stephen J. Taerum ${ }^{1}$, Michael J. Wingfield ${ }^{1}$, XuDong Zhou $^{1}$ and Z. Wilhelm de Beer $^{1^{*}}$ (ID

\begin{abstract}
Bark beetle galleries are complex ecosystems where many microbes and other arthropods co-exist with the beetles. Fungi isolated from these galleries are often referred to as 'beetle associates', but the nature of these associations are poorly understood. The possibility that many of these fungi might in fact be mite associates is often overlooked. Several recent studies explored the diversity of fungi from conifer-infesting bark beetles and their galleries in China, but only one study considered phoretic mites and their fungi from conifer-infesting bark beetles in Yunnan, southwestern China. We studied the mites and fungi from galleries of four spruce-infesting bark beetle species in the high altitude forests of Qinghai province, western China. Mites were identified based on morphological characteristics, and fungi based on DNA sequences of four gene regions. In total, 173 mite individuals were collected belonging to 18 species in 11 genera. A total of 135 fungal isolates were obtained from the mites, representing 14 taxa from the Ophiostomatales. The most frequently isolated fungus was Ophiostoma nitidum, which represented $23.5 \%$ of the total isolates. More fungal species were found from fewer mites and bark beetle species than from the study in Yunnan. Although we could not elucidate the exact nature of interactions between mites and their fungi, our results re-enforce that these organisms should not be ignored in pest risk assessments of bark beetles, that often focus only on the beetles and their fungi. Three new species are described: Grosmannia zekuensis, $O$. manchongi, and $O$. kunlunense spp. nov., and our data revealed that $O$. typographi, recently described from China, is a synonym of $O$. ainoae.
\end{abstract}

Keywords: Spruce, Symbiosis, Ascomycetes, Scolytinae, Three new taxa

\section{INTRODUCTION}

Mites (Arachnida, Acari) are commonly associated with bark beetles (Insecta, Coleoptera, Scolytinae) and their galleries (Hofstetter et al. 2015). Because of their small size, these mite species rely on the bark beetles for dispersal between plant hosts (Hofstetter et al. 2013). To date, about 270 mite species have been identified as associates of a limited number of bark beetle species, and many more are likely to be discovered (Hofstetter et al. 2013, 2015).

\footnotetext{
* Correspondence: wilhelm.debeer@fabi.up.ac.za

'Department of Biochemistry, Genetics \& Microbiology, Forestry and Agricultural Biotechnology Institute (FABI), University of Pretoria, Pretoria 0002, South Africa

Full list of author information is available at the end of the article
}

The ecological roles of very few bark beetle-associated mites are well understood, but the different classes of feeding behavior (Hofstetter et al. 2013) suggests that their impacts in gallery ecosystems could be broad. Some of these mites, such as Pyemotes dryas, predate upon or parasitize the eggs and early larval instars of bark beetles (Wegensteiner et al. 2015) and thus have a direct effect on population dynamics of the beetles. However, the impacts of many mites on bark beetle behavior and population growth are indirect. A few species are known to be predators of nematodes, while many of the mite species are omnivores and feed on fungi, nematodes and dead arthropods in the galleries. Other species

(c) The Author(s). 2020 Open Access This article is licensed under a Creative Commons Attribution 4.0 International License, which permits use, sharing, adaptation, distribution and reproduction in any medium or format, as long as you give appropriate credit to the original author(s) and the source, provide a link to the Creative Commons licence, and indicate if changes were made. The images or other third party material in this article are included in the article's Creative Commons licence, unless indicated otherwise in a credit line to the material. If material is not included in the article's Creative Commons licence and your intended use is not permitted by statutory regulation or exceeds the permitted use, you will need to obtain permission directly from the copyright holder. To view a copy of this licence, visit http://creativecommons.org/licenses/by/4.0/. 
are exclusively mycetophagous, utilizing fungi as their only source of nutrition (Hofstetter et al. 2013).

It appears that some mycetophagous mites have preference for one or a few specific species of fungi. One such an example is Tarsonemus krantzi, associated with the southern pine beetle, Dendroctonus frontalis, that vector and feeds on Ophiostoma minus (Lombardero et al. 2000). Other mites such as Histiogaster spp. are generalists that can feed on several different fungal species (Hofstetter and Moser 2014). The most common fungi associated with bark beetles and their mites are the ophiostomatoid fungi (Hofstetter and Moser 2014). This is a polyphyletic group of fungi that includes several genera in Microascales and Ophiostomatales, that are characterized by spores produced in sticky droplets to facilitate dispersal by bark beetles and mites (De Beer et al. 2013). Many bark beetles have specialized structures known as mycangia in which to carry the spores of these fungi (Six 2012). In the case of mites, most fungi appear to be vectored on their exterior surfaces (Moser et al. 1989b), but some mite species have evolved specialized structures known as, sporothecae, to ensure the successful transmission of specific fungal symbionts between hosts (Moser 1985).

Although the impact of most fungi on the fitness of the mites or their beetle vectors remains largely unknown, the three-way interaction between beetles, mites and fungi, has been elucidated in the case of the southern pine beetle, Dendroctonus frontalis, and its associated Tarsonemus mites. The larvae of $D$. frontalis are obligately dependent on two fungal species, Ceratocystiopsis ranaculosus and Entomocorticium sp. A, for nutrition (Ayres et al. 2000). These fungi are outcompeted by Ophiostoma minus (Barras 1970; Bridges 1983; Goldhammer et al. 1990), the nutritional fungal mutualist of Tarsonemus mites, when the mites are present in the galleries of $D$. frontalis (Hofstetter et al. 2013). As a result of these interactions, large populations of Tarsonemus mites can suppress the reproduction of $D$. frontalis (Hofstetter et al. 2006).

The relationships between mites and their beetle vectors are generally unspecific, and a single species of mite can use many different beetle species as vector in order to reach its tree hosts (Hofstetter et al. 2013). Similarly, many ophiostomatoid fungi are promiscuous in their association with beetle species, and a single beetle species can vector a number of different fungal species (Kirisits 2004; Linnakoski et al. 2012; Taerum et al. 2013). In addition, one mite species can carry any of a number of ophiostomatoid fungi (Chang et al. 2017). It thus becomes extremely difficult to resolve specificity between any of these organisms. However, where galleries of different bark beetle species might be restricted to different parts of a specific tree and thus not overlap, it has been suggested that mites might facilitate the movement of fungal species between galleries of different beetle species (Chang et al. 2017).

There are several serious tree pathogens amongst the ophiostomatoid fungi. These include the well-known Dutch Elm Disease fungi, Ophiostoma ulmi and two varieties of O. novo-ulmi (Brasier 1990, 1991). These pathogens are vectored by Scolytus bark beetles (Webber 2004), but it has been shown that phoretic mites also vector the pathogens and might contribute to the spore load and the high efficiency of $S$. scolytus in spreading Dutch elm disease (Moser et al. 2010). Leptographium wingfieldii is a mildly pathogenic ophiostomatoid fungus vectored by the pine shoot beetle, Tomicus piniperda, in its native range in Europe (Solheim 1991). The beetle was first detected in the USA in the early 1990's, and it was later shown that $L$. wingfieldii was introduced with the beetle, and that the fungus has subsequently became an associate of two native north American beetles, Dendroctonus valens and Ips pini (Jacobs et al. 2004). Although the mechanism by which $L$. wingfieldii was transferred between the beetle species was not considered (Jacobs et al. 2004), it is likely that mites facilitated the transfer. Such novel associations between ophiostomatoid fungi, bark- or ambrosia beetles and trees, can pose serious threats to both natural and commercial forests, as well as tree crops. The role of mites in the establishment of these associations are poorly studied, most probably underestimated, and can only be understood if their associations with fungi are explored in natural ecosystems.

The taxonomy of Ophiostomatales was revised by De Beer \& Wingfield (2013) who considered all published ribosomal large subunit (LSU) and internal transcribed spacer (ITS) sequences. They recognized six genera and 18 species complexes in the order, including Ophiostoma, Raffaelea, Ceratocystiopsis, Fragosphaeria, Graphilbum, and Leptographium sensu lato. In a subsequent paper De Beer et al. (2016a) elevated the $S$. schenckii - O. stenoceras complex to genus level, and re-instated the name Sporothrix for this group. Three smaller, novel genera had also been described recently in the order, Hawksworthiomyces (De Beer et al. 2016b), Aureovirgo (Van Der Linde et al. 2016) and Afroraffaelea (Bateman et al. 2017). For the purpose of the present study, we define Ophiostoma sensu stricto in agreement with De Beer et al. (2016a), that then includes the O. ulmi-, O. piceae-, O. ips-, and O. clavatum species complexes. A number of Ophiostoma species group outside Ophiostoma s. str., and their position remain unresolved. For the present they are included next to Ophiostoma s. str. in a more loosely defined Ophiostoma sensu lato. Leptographium s. lat. incorporates Leptographium s. str. and the Grosmannia penicillata complex as defined by De Beer and Wingfield (2013) and Yin et al. (2020). 
To date, the majority of studies on interactions between fungi, bark beetles and mites have been conducted on Dendroctonus, Ips and Dryocoetes spp. in North America (Klepzig and Hofstetter 2011; Hofstetter et al. 2013, 2015; Hofstetter and Moser 2014), and Scolytus, Ips and Pityokteines spp. in Europe (Levieux et al. 1989; Moser et al. 1989a, 2005, 2010; Linnakoski et al. 2016b). In southern Africa, ongoing studies focus on the interactions between mites and ophiostomatoid fungi in infructescences of Protea spp. (Roets et al. 2007, 2009, 2011). There have been only two studies reporting on the fungal associates of mites form east Asia. In these cases, Moser et al. (1997) reported on the fungal associates of mites on Ips typographus in Japan, and Chang et al. (2017) described fungi from mites associated with various conifer-infesting bark beetles in Yunnan, China.

In recent years, 98 ophiostomatoid species, including 49 new species, have been reported in association with bark beetles from China (Table S1) (Lu et al. 2009a, 2009b; Paciura et al. 2010a, 2010b; Zhou et al. 2011, 2013; Taerum et al. 2013; Yin et al. 2015, 2016, 2019, 2020; Wang et al. 2016, 2018, 2019, 2020; Chang et al. 2017, 2019; Liu et al. 2017). The study by Chang et al. (2017) was the first to report ophiostomatoid fungi associated with phoretic mites. They reported 11 species from mites associated with bark beetles infesting Pinus kesiya, of which four were described as new species. Three of the studies from China, included isolates from Qinghai province (Yin et al. 2016, 2019, 2020). This province, which is located on the Qinghai-Tibetan Plateau, is one of the world's biodiversity hotspots because of its diverse landscapes, and complex geological and climatic history (Li et al. 2012). Yin et al. (2016) decribed five new Ophiostoma spp. from four spruce-infesting bark beetles, two of which are Ips spp. that cause severe damage to spruce trees are in this area (Liu et al. 2008). In addition, two Leptographium (Yin et al. 2019) and four Grosmannia spp. (Yin et al. 2020) were described from Polygraphus poligraphus and Ips shangrila, also attacking spruce. Apart from these 11 species, no other ophiostomatoid fungi have been reported from Qinghai. Furthermore, nothing is known regarding the fungal associates of phoretic mites on spruce-infesting beetles in China. In this study, we addressed the following questions: 1) which mite species are associated with spruceinfesting bark beetles in Qinghai, and 2) which species of ophiostomatoid fungi are associated with these mites?

\section{MATERIAL AND METHODS}

\section{Collection of mites and fungi}

A survey was conducted on Picea crassifolia and Picea purpurea in July 2010, during the flight period of bark beetles, in the Maixiu and Xianmin forest farms in Qinghai province, China. Bark beetle galleries were collected and stored in re-sealable plastic bags at $4{ }^{\circ} \mathrm{C}$ until isolations could be made. Living mites were collected from the galleries under a dissecting microscope. Each individual mite was placed on a separate Petri dish containing malt extract agar (MEA, 20 g Difico agar, 20 g Difico BactoTM malt extract [Becton, Dickinson \& Company], $1 \mathrm{~L}$ deionized water) medium. After the plates were sealed, the mites were allowed to crawl over the plates for $24 \mathrm{~h}$. The mites were then removed and stored in $1.5 \mathrm{~mL}$ Eppendorf tubes containing 75\% Ethanol for later identification by Dr. E.A. Ueckermann (Plant Protection Research Institute, Agricultural Research Council, South Africa).

The MEA plates were incubated at $20^{\circ} \mathrm{C}$ until fungal growth was evident. The hyphal tips of colonies were transferred to fresh MEA plates to obtain pure cultures. All isolates used in this study were deposited into the Culture Collection (CMW) of the Forestry and Agricultural Biotechnology Institute (FABI), University of Pretoria, Pretoria, Republic of South Africa. Isolates representing types of new species were deposited in the culture collection (CBS) of the Westerdijk Fungal Biodiversity Institute, Utrecht, the Netherlands.

\section{DNA sequencing and phylogenetic analyses}

Isolates were grown on 2\% MEA medium. DNA was extracted using PrepMan ultra sample preparation reagent (Applied Biosystems, Foster City, CA) following the manufacturer's recommendations. The internal transcribed spacer regions 1 and 2 (ITS), including the $5.8 \mathrm{~S}$ region, were amplified using the primers ITS1F and ITS4 (White et al. 1990; Gardes and Bruns 1993), the $\beta$-tubulin (BT) gene was amplified using the primer pair of $\mathrm{Bt} 2 \mathrm{a}$ and $\mathrm{Bt} 2 \mathrm{~b}$ (Glass and Donaldson 1995), and the elongation factor 1$\alpha(E F)$ gene was amplified using the primer pair of EF2F (Marincowitz et al. 2015) and EF2R (Jacobs et al. 2004). In addition, the nuclear large subunit (LSU) was amplified with the primer pair LR0R and LR5 (Vilgalys and Hester 1990) for fungi that reside in Leptographium sensu lato. PCR and sequencing were conducted following the protocols described by Duong et al. (2012).

The sequences obtained with the forward and reverse primers were aligned and contigs constructed using the program Geneious pro v. 7.1.4 (Biomatters, Auckland, New Zealand). All sequences obtained in this study were deposited in GenBank. BLAST searches of the ITS sequences were conducted in NCBI GenBank for preliminary identifications. Based on the BLAST results, sequence data for other markers were separated according to the relevant species complexes. For taxa residing in Leptographium s. lat., the ITS2-LSU regions were used to determine generic placement, unlike the case for Ophiostoma spp. where the ITS1-ITS2 regions were used for this purpose. The $B T$ and $E F$ data sets were analyzed separately for each species complex. Alignments were made using an 
online version of MAFFT v. 7 with default settings (Katoh and Standley 2013). All aligned sequence datasets were submitted to TreeBase (No. 24829).

Phylogenetic analyses including maximum likelihood (ML), maximum parsimony (MP) and Bayesian inference (BI) were conducted for all datasets. The best substitution models for each data set were determined using jModelTest v. 2.1.6 (Darriba et al. 2012) on the CIPRES Science Gateway v. 3.3 (Miller et al. 2010). ML analyses were conducted using RaxML v. 8.2.4 on the CIPRES Science Gateway v. 3.3 (Stamatakis 2014) with default GTR substitution matrix and 1000 rapid bootstraps. MP analyses were performed using PAUP v. 4.0b10 (Swofford 2002), gaps were treated as a fifth character. BI analyses were conducted using MrBayes v. 3.2.6 (Ronquist et al. 2012) on the CIPRES Science Gateway v. 3.3. Four MCMC chains were run from a random starting tree for 5 million generations and trees were sampled every 100th generation. $25 \%$ of trees sampled were discarded as burn-in and the remaining trees were used to construct majority rule consensus trees.

\section{Growth studies}

Mycelium-covered agar plugs were transferred from the actively growing margins of one-week-old cultures and placed at the centers of $90 \mathrm{~mm}$ Petri dishes containing $2 \%$ MEA. The optimal temperatures for growth were determined at temperatures ranging from 5 to $35^{\circ} \mathrm{C}$ at $5{ }^{\circ} \mathrm{C}$ intervals and there were three replicate plates for each temperature. Cultures were incubated in the dark. Colony diameters were measured every 2 days until hyphae reached the edges of the Petri dishes, at which point the temperatures for optimum growth were noted.

\section{Morphological studies}

Asexual and/or sexual structures were mounted in lactophenol on glass slides, covered with coverslips and examined with a Zeiss Axioskop 2 Plus compound microscope or a Zeiss Discovery V12 dissection microscope with an Axiocam digital camera (Axiovision 3.1) (München-Hallbergmoos, Germany). Fifty measurements were made for each taxonomically informative structure. The measurements were given in the format (minimum-) mean minus standard deviation-mean plus standard deviation (-maximum).

\section{Frequency of isolation}

The following formula was used to calculate the frequencies of isolation of the ophiostomatoid species: $\mathrm{F}=(\mathrm{NF} / \mathrm{NT}) \times 100$, where $\mathrm{F}$ represented the frequency of isolation (\%), NT represented the total number of isolates obtained, and NF represented the number of isolates for each particular taxon.

\section{RESULTS}

\section{Collection of mites and fungi}

In total, 173 mite individuals representing 18 mite species residing in 11 genera were collected from galleries of four bark beetle species on two host tree species (Table 1). The bark beetles were Dendroctonus micans, Ips shangrila, Ips nitidus and Polygraphus poligraphus. All D. micans, I. nitidus and P. poligraphus were collected from Pi. crassifolia, and I. shangrila was collected from Pi. purpurea. Three mite species were associated with $D$. micans, 13 mite species with $I$. nitidus, five mite species with $I$. shangrila, and three mite species with $P$. poligraphus. Most mite species were found only in the galleries of a single bark beetle species. Exceptions were for Insectolaelaps sp. 1, Uropodoidea sp. 4, Uropodoidea sp. 6, and Winterschmidtiidae sp., which were found in the galleries of more than one beetle species (Table S2).

In total, 135 ophiostomatoid fungal isolates were obtained from 65 mite individuals (Table S3). Seventy isolates were collected from 13 mite species in galleries of I. nitidus, 33 isolates were collected from three mite species in galleries of D. micans, 19 isolates were collected from five mite species in galleries of $I$. shangrila, and 13 isolates were collected from three mite species in $P$. poligraphus. Twenty-eight isolates were collected from one mite species in the family Winterschmidtiidae and 23 isolates collected from Uropodoidea sp. 4. More than 10 isolates were collected from each of Insectolaelaps sp. 2, Insectolaelaps sp. 1 and Uropodoidea sp. 6.

\section{DNA sequencing and phylogenetic analysis}

Based on analysis of ITS and ITS-LSU sequence data, of the total 135 isolates collected in this study, 87 isolates resided in Ophiostoma sensu stricto (Fig. 1), and the remaining 48 isolates resided in Leptographium s. lat. (Fig. 2). Most of the isolates belonging to Ophiostoma s. str. resided in three species complexes namely the $O$. piceae-, O. clavatum- and O. ips species complexes, while most of the isolates belonging to Leptographium s. lat. resided in the Grosmannia penicillata species complex. Based on the availability of sequence data from previously studies, datasets of different protein coding gene regions were compiled and analysed separately for different species complexes. Phylogenetic analyses of these datasets separated the isolates into 14 distinct taxa (Table 2), 11 of which belonged to previously described species and three represented novel species.

In Ophiostoma s. str., two taxa (Taxa 1 and 4) represented by four and three isolates respectively, grouped peripheral to the O. piceae complex in the ITS tree (Fig. 1). Analyses of $B T$ and $E F$ sequences data (Fig. 3) showed that Taxon 1 belong to $O$. tetropii, and isolates of Taxon 4 formed a distinct, well supported clade (with 100\% ML bootstrap support and BI posterior probabilities larger than 0.9 for both 
Table 1 Mites (Acari) collected from bark beetle galleries on spruce in Qinghai in this study

\begin{tabular}{|c|c|c|c|c|c|c|}
\hline Mite & Family Name & Species Name & $\mathrm{NI}{ }^{\mathbf{a}}$ & $\mathrm{NI} / \mathrm{TN}^{\mathbf{b}}$ & $\mathrm{NMCF}^{\mathrm{c}}$ & $\mathrm{NMCF} / \mathrm{NI}{ }^{\mathrm{d}}$ \\
\hline $\bar{M} 1$ & Acaridae & Horstia sp. & 1 & 0.0061 & 0 & 0.000 \\
\hline M 2 & Acaridae & Schwiebea wainsteini & 2 & 0.0121 & 2 & 1.000 \\
\hline M 3 & Ascidae & Diseius cf. ulmi & 3 & 0.0182 & 1 & 0.333 \\
\hline M 4 & Digamasellidae & Dendrolaelaps sp. & 1 & 0.0061 & 0 & 0.000 \\
\hline M 5 & Digamasellidae & Insectolaelaps sp. 2 & 23 & 0.1394 & 7 & 0.304 \\
\hline M 6 & Digamasellidae & Insectolaelaps sp. 1 & 15 & 0.0909 & 7 & 0.467 \\
\hline M 7 & Ereynetidae & Ereynetes sp. & 3 & 0.0182 & 2 & 0.667 \\
\hline M 8 & Erynetidae & sp. & 1 & 0.0061 & 0 & 0.000 \\
\hline M 9 & Unknown & sp. 1 & 6 & 0.0364 & 1 & 0.167 \\
\hline M 10 & Melicharidae & Proctolaelaps nr. hystrix & 10 & 0.0606 & 5 & 0.500 \\
\hline M 11 & Mesostigmata & sp. 1 & 1 & 0.0061 & 1 & 1.000 \\
\hline M 12 & Mesostigmata & sp. 2 & 1 & 0.0061 & 0 & 0.000 \\
\hline M 13 & Mesostigmata & sp. 3 & 1 & 0.0061 & 1 & 1.000 \\
\hline M 14 & Mesostigmata & sp. 4 & 2 & 0.0121 & 1 & 0.500 \\
\hline M 15 & Unknown & sp. 2 & 6 & 0.0364 & 1 & 0.167 \\
\hline M 16 & Pygmephoridae & Bakerdania sp. & 5 & 0.0303 & 1 & 0.200 \\
\hline M 17 & Tarsonemidae & Tarsonemus sp. & 7 & 0.0424 & 1 & 0.143 \\
\hline M 18 & Uropodoidea & sp. 4 & 35 & 0.2121 & 13 & 0.371 \\
\hline M 19 & Uropodoidea & sp. 5 & 1 & 0.0061 & 0 & 0.000 \\
\hline M 20 & Uropodoidea & sp. 6 & 35 & 0.2121 & 9 & 0.257 \\
\hline M 21 & Uropodoidea & sp. 7 & 1 & 0.0061 & 1 & 1.000 \\
\hline M 22 & Uropodoidea & sp. 8 & 1 & 0.0061 & 0 & 0.000 \\
\hline M 23 & Winterschmidtiidae & sp. & 11 & 0.0667 & 10 & 0.909 \\
\hline M 24 & Zerconidae & Zercon sp. & 1 & 0.0061 & 1 & 1.000 \\
\hline Total & & & 173 & & 65 & 0.376 \\
\hline
\end{tabular}

${ }^{\mathrm{a}} \mathrm{NI}$ Number of mite individuals

${ }^{b}$ NI/TN Number of mite individuals/Total number of mite individuals

c NMCF Number of mites carrying fungi

${ }^{\mathrm{d}}$ NMCF/NI Number of mites carrying fungi/ Number of mite individuals

$B T$ and $E F$ gene regions) which was separated from all previously described species, and thus this taxon represented a novel species. Taxa 2 and 3, represented by 32 and 11 isolates respectively, grouped with $O$. nitidus and $O$. qinghaiense in O. piceae complex. Taxa 5 and 6 grouped in the O. clavatum complex (Fig. 1) and were represented by ten and 11 isolates respectively. Based on the $B T$ and $E F$ sequence data (Fig. S1) the Taxon 5 isolates grouped in a single clade with the ex-type isolates of both $O$. ainoae and $O$. poligraphi, suggesting that these represented a single species. Taxon 6 isolates grouped with sequences of $O$. shangrilae. Taxon 7 resided close to and Taxon 8 within the $O$. ips complex (Fig. 1) and were represented by ten and six isolates respectively. Based on ITS and BT data (Fig. 4) isolates of Taxon 7 formed a distinct and well supported clade (with 97\% ML bootstrap support for ITS, 100\% ML bootstrap support for $B T$, and BI posterior probabilities larger than 0.9 for both ITS and $B T$ gene regions) which was closest to, but clearly distinct from $O$. japonicum, and thus this taxon represented a novel species. Taxon 8 isolates grouped in a monophyletic lineage with several isolates of $O$. bicolor.

In Leptographium s. lat., Taxon 9 represented by only one isolate grouped in Group A of Leptographium s. lat. together with $L$. pineti and $L$. ningerensis (Fig. 2) and BT and $E F$ sequence analyses confirmed this Taxon was conspecific with L. ningerensis (Fig. S2). Taxon 10 grouped in the L. olivaceum complex (Fig. 2) and $B T$ and $E F$ sequence analyses confirmed that this isolate represented L. breviuscapum (Fig. S2). Taxon 11 grouped peripheral to the G. penicillata complex with $L$. taigense (Fig. 2) and $B T$ and $E F$ sequences analyses confirmed the identity of the species as L. taigense (Fig. 5). Taxa 12, 13, and 14 grouped in the G. penicillata complex (Fig. 2) and based on $B T$ and EF sequences (Fig. 5), Taxon 12 was identified as G. purpurea, and Taxon 14 was identified as G. xianmiense, while isolates of Taxon 13 formed a distinct and well supported clade (with ML bootstrap supports larger than 95\%, and BI posterior probabilities larger than 0.9 for both $B T$ anf $E F$ gene regions), 


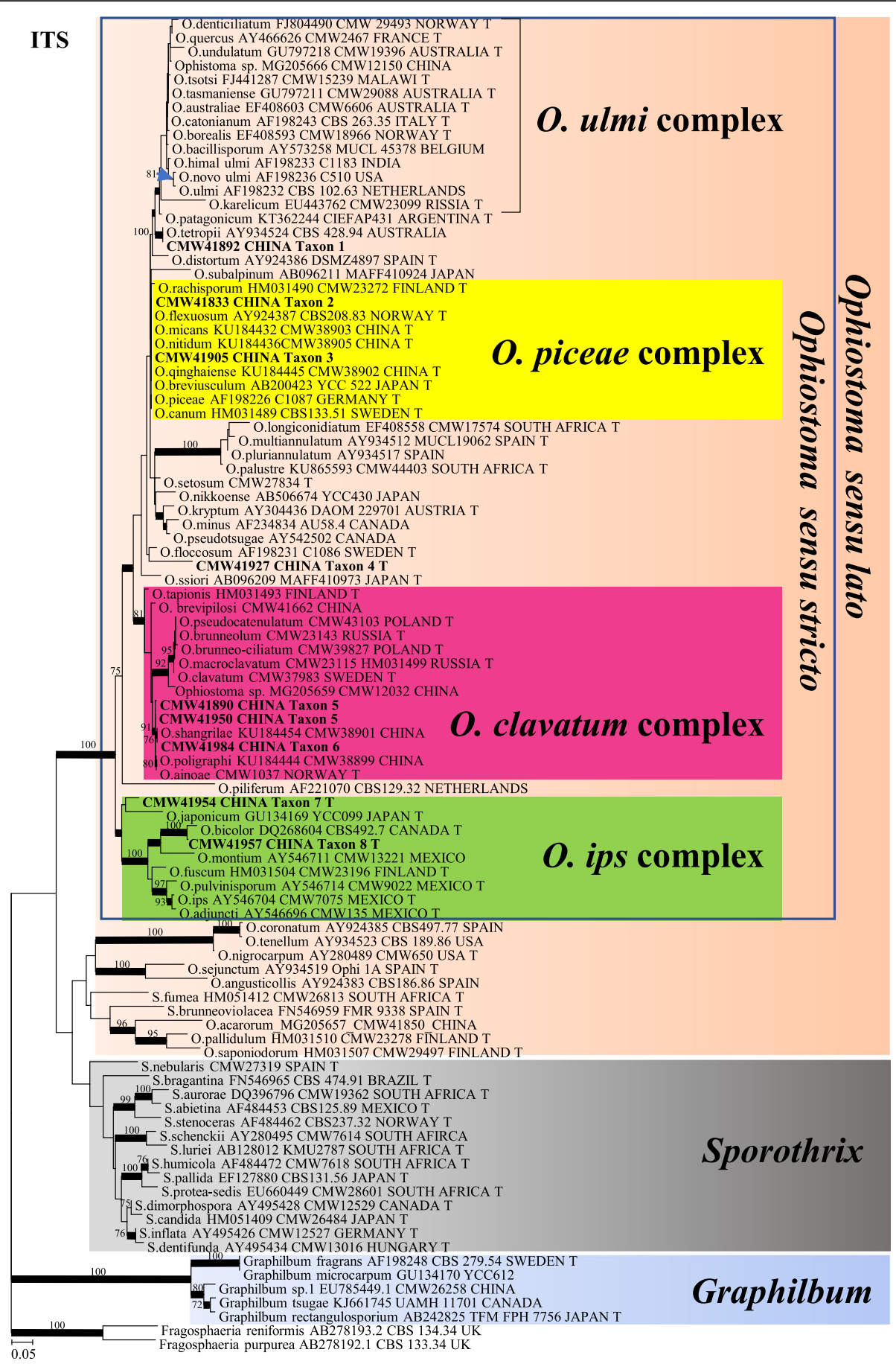

Fig. 1 Phylogram obtained from ML analyses of the ITS region of Ophiostoma. Sequences obtained in this study are printed in bold type. ML bootstrap support values (1000 replicates, normal type) above 75\% are indicated at the nodes. Posterior probabilities (above 0.9) obtained from BI are indicated by bold lines at the relevant branching points. $T=$ ex-type cultures. Scale bar = total nucleotide difference between taxa

which was close to but different from G. purpurea, thus this taxon represented a novel species.

\section{Frequencies of isolation}

The most frequently collected mite species were Uropodoidea sp. 4 and Uropodoidea sp. 6 each of which represented $21.2 \%$ of all mites (Table 1 ). The next most frequently collected species was Insectolaelaps sp. 2 which represented $13.9 \%$ of the mites. A few species were collected at very low frequencies, such as Uropodoidea sp. 7 and Uropodoidea sp. 8 that only represented $0.6 \%$ of the collections. 


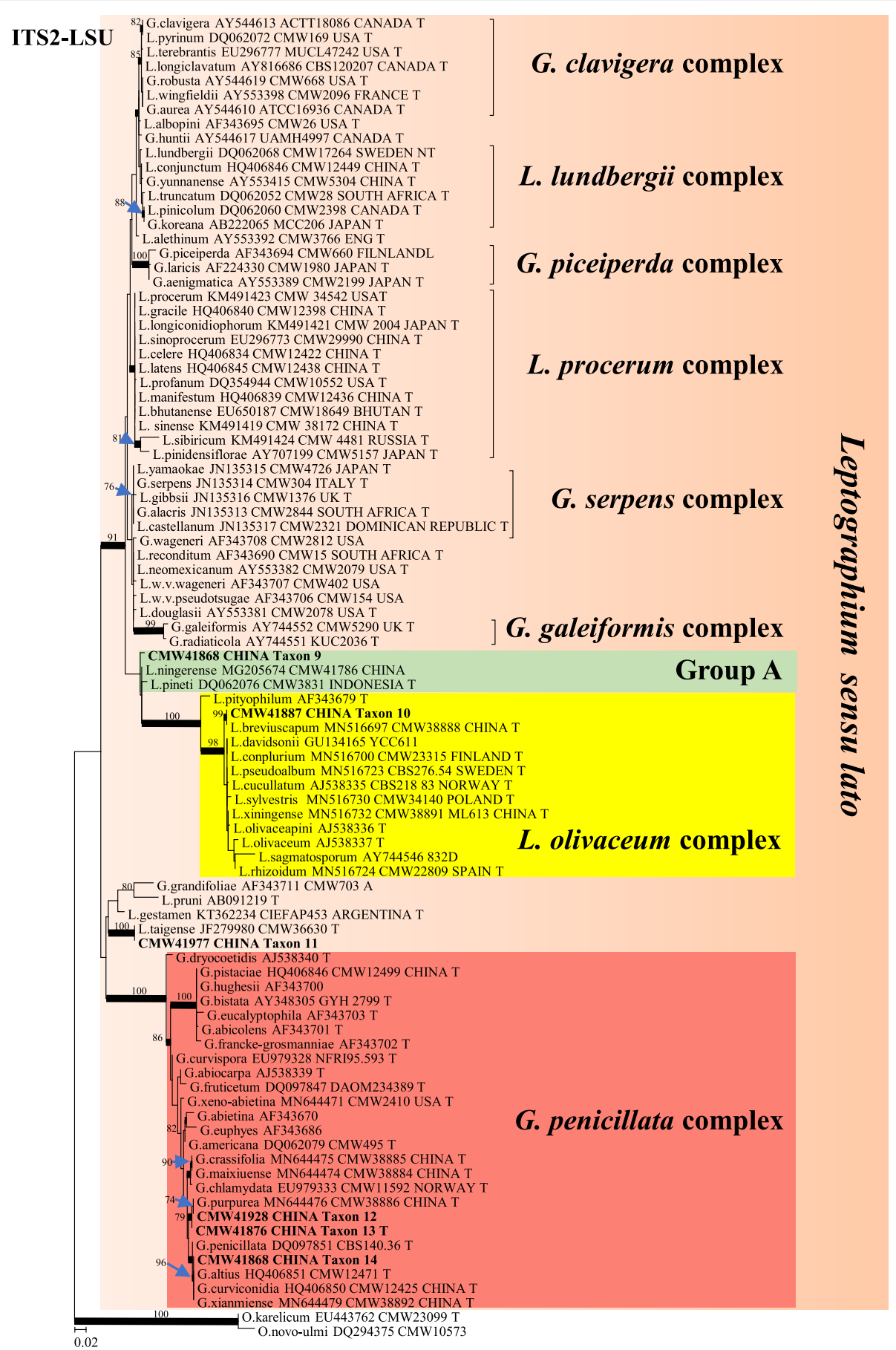

Fig. 2 Phylogram obtained from ML analyses of the ITS2-LSU region of Leptographium. Sequences obtained in this study are printed in bold type. ML bootstrap support values (1000 replicates, normal type) above 75\% are indicated at the nodes. Posterior probabilities (above 0.9) obtained from $\mathrm{BI}$ are indicated by bold lines at the relevant branching points. $\mathrm{T}=$ ex-type cultures. Scale bar = total nucleotide difference between taxa

The most frequently isolated ophiostomatoid fungi were O. nitidum (Taxon 2) and L. taigense (Taxon 11), which represented 23.5 and $22.8 \%$ of the total isolates respectively (Table S3). This was followed by $O$. qinghaiense (Taxon 3, 8.1\%) and O. shangrilae (Taxon 6, 8.1\%), and $O$. ainoae (Taxon 5, 7.4\%) and Taxon 8 (7.4\%). The fungi with the lowest frequency of isolations were L. breviuscapum (Taxon 10) and L. ningerensis (Taxon 9), both of which represented $0.7 \%$ of total isolates. The remaining fungi were found at frequencies lower than $5 \%$. 
Table 2 Isolates of ophiostomatoid fungi obtained from different mites in Qinghai. Species names of novel taxa are printed in bold type

\begin{tabular}{|c|c|c|c|c|c|c|c|c|c|c|}
\hline \multirow[t]{2}{*}{ Taxon } & \multirow[t]{2}{*}{ Species } & \multicolumn{2}{|c|}{$\begin{array}{l}\text { Isolate } \\
\text { number }\end{array}$} & \multirow[t]{2}{*}{ Host } & \multirow[t]{2}{*}{ Beetle } & \multirow[t]{2}{*}{ Mite $^{c}$} & \multirow[t]{2}{*}{ Locations } & \multicolumn{3}{|c|}{ GenBank number $^{\mathbf{d}}$} \\
\hline & & CMW & CBS & & & & & $\begin{array}{l}\text { ITS/ITS2- } \\
\text { LSU }\end{array}$ & $B T$ & $E F$ \\
\hline \multirow[t]{4}{*}{1} & Ophiostoma tetropii & 41891 & & $\begin{array}{l}\text { Picea } \\
\text { crassifolia }\end{array}$ & Dendroctonus micans & M11 & Maixiu & MH121623 & MH124426 & $\mathrm{MH} 124490$ \\
\hline & & 41892 & & P. crassifolia & D. micans & M11 & Maixiu & MH121624 & MH124427 & MH124491 \\
\hline & & 41893 & & P. crassifolia & D. micans & M23 & Maixiu & MH121625 & MH124428 & MH124492 \\
\hline & & 41938 & & P. crassifolia & Ips nitidus & M7 & Xianmi & MH121626 & MH124429 & MH124493 \\
\hline \multirow[t]{15}{*}{2} & O. nitidum & 41883 & & P. crassifolia & $\begin{array}{l}\text { Polygraphus } \\
\text { poligraphus }\end{array}$ & M6 & Maixiu & MH121627 & MH124430 & $\mathrm{MH} 124494$ \\
\hline & & 41874 & & P. crassifolia & I. nitidus & M9 & Maixiu & MH121628 & $\mathrm{MH} 124431$ & $\mathrm{MH} 124495$ \\
\hline & & 41886 & & P. crassifolia & P. poligraphus & M6 & Maixiu & MH121629 & MH124432 & MH124496 \\
\hline & & 41895 & & P. crassifolia & D. micans & M23 & Maixiu & MH121630 & MH124433 & $\mathrm{MH} 124497$ \\
\hline & & 41898 & & P. crassifolia & D. micans & M3 & Maixiu & MH121631 & MH124434 & MH124498 \\
\hline & & 41899 & & P. crassifolia & D. micans & M3 & Maixiu & MH121632 & MH124435 & MH124499 \\
\hline & & 41901 & & P. crassifolia & D. micans & M23 & Maixiu & MH121633 & MH124436 & MH124500 \\
\hline & & 41902 & & P. crassifolia & D. micans & M23 & Maixiu & MH121634 & MH124437 & $\mathrm{MH} 124501$ \\
\hline & & 41911 & & P. crassifolia & D. micans & M23 & Maixiu & MH121635 & MH124438 & MH124502 \\
\hline & & 41917 & & P. crassifolia & D. micans & M23 & Maixiu & MH121636 & MH124439 & MH124503 \\
\hline & & 41918 & & P. crassifolia & D. micans & M23 & Maixiu & MH121637 & MH124440 & MH124504 \\
\hline & & 41923 & & Picea purpurea & Ips shangrila & M6 & Maixiu & MH121638 & MH124441 & MH124505 \\
\hline & & 41933 & & P. purpurea & I. shangrila & M17 & Maixiu & MH121639 & MH124442 & MH124506 \\
\hline & & 41934 & & P. purpurea & I. shangrila & M17 & Maixiu & MH121640 & MH124443 & MH124507 \\
\hline & & 41939 & & P. crassifolia & I. nitidus & M20 & Xianmi & MH121641 & $\mathrm{MH} 124444$ & MH124508 \\
\hline \multirow[t]{6}{*}{3} & O. qinghaiense & 41900 & & P. crassifolia & D. micans & M3 & Maixiu & MH121642 & MH124445 & MH124509 \\
\hline & & 41903 & & P. crassifolia & D. micans & M23 & Maixiu & MH121643 & MH124446 & MH124510 \\
\hline & & 41905 & & P. crassifolia & D. micans & M23 & Maixiu & MH121644 & MH124447 & MH124511 \\
\hline & & 41906 & & P. crassifolia & D. micans & M23 & Maixiu & MH121645 & MH124448 & $\mathrm{MH} 124512$ \\
\hline & & 41907 & & P. crassifolia & D. micans & M23 & Maixiu & MH121646 & MH124449 & MH124513 \\
\hline & & 41915 & & P. crassifolia & D. micans & M23 & Maixiu & MH121647 & $\mathrm{MH} 124450$ & $\mathrm{MH} 124514$ \\
\hline \multirow[t]{3}{*}{4} & O. kunlunense & 41927 & $141903^{\mathrm{H}}$ & P. purpurea & I. shangrila & M19 & Maixiu & MH121648 & MH124451 & MH124515 \\
\hline & & 48853 & 141904 & P. purpurea & I. shangrila & M6 & Maixiu & MH121649 & MH124452 & $\mathrm{MH} 124516$ \\
\hline & & 48854 & 141905 & P. purpurea & 1. shangrila & M18 & Maixiu & MH121650 & MH124453 & - \\
\hline \multirow[t]{5}{*}{5} & O. ainoae & 41882 & & P. crassifolia & P. poligraphus & M20 & Maixiu & MH121651 & MH124454 & MH124517 \\
\hline & & 41890 & & P. crassifolia & P. poligraphus & M14 & Maixiu & MH121652 & MH124455 & MH124518 \\
\hline & & 41958 & & P. crassifolia & I. nitidus & M18 & Xianmi & MH121653 & MH124456 & $\mathrm{MH} 124519$ \\
\hline & & 41881 & & P. crassifolia & P. poligraphus & M20 & Maixiu & MH121654 & $\mathrm{MH} 124457$ & $\mathrm{MH} 124520$ \\
\hline & & 41950 & & P. crassifolia & I. nitidus & M10 & Xianmi & MH121655 & MH124458 & MH124521 \\
\hline \multirow[t]{5}{*}{6} & O. shangrilae & 41885 & & P. crassifolia & P. poligraphus & M6 & Maixiu & MH121656 & MH124459 & MH124522 \\
\hline & & 41930 & & P. purpurea & I. shangrila & M18 & Maixiu & MH121657 & MH124460 & MH124523 \\
\hline & & 41968 & & P. crassifolia & I. nitidus & M24 & Xianmi & MH121658 & MH124461 & MH124524 \\
\hline & & 41983 & & P. crassifolia & I. nitidus & M5 & Xianmi & MH121659 & MH124462 & MH124525 \\
\hline & & 41984 & & P. crassifolia & I. nitidus & M5 & Xianmi & MH121660 & MH124463 & MH124526 \\
\hline \multirow[t]{2}{*}{7} & O. manchongi & 41872 & & P. crassifolia & I. nitidus & M10 & Maixiu & MH121661 & MH124464 & $\mathrm{MH} 124527$ \\
\hline & & 41954 & $141906^{\mathrm{H}}$ & P. crassifolia & I. nitidus & M18 & Xianmi & MH121662 & MH124465 & - \\
\hline
\end{tabular}


Table 2 Isolates of ophiostomatoid fungi obtained from different mites in Qinghai. Species names of novel taxa are printed in bold type (Continued)

\begin{tabular}{|c|c|c|c|c|c|c|c|c|c|c|}
\hline \multirow[t]{2}{*}{ Taxon } & \multirow[t]{2}{*}{ Species } & \multicolumn{2}{|c|}{$\begin{array}{l}\text { Isolate } \\
\text { number }{ }^{\mathbf{a}, \mathbf{b}}\end{array}$} & \multirow[t]{2}{*}{ Host } & \multirow[t]{2}{*}{ Beetle } & \multirow[t]{2}{*}{ Mite $^{c}$} & \multirow[t]{2}{*}{ Locations } & \multicolumn{3}{|c|}{ GenBank number ${ }^{\mathbf{d}}$} \\
\hline & & CMW & CBS & & & & & $\begin{array}{l}\text { ITS/ITS2- } \\
\text { LSU }\end{array}$ & $B T$ & $E F$ \\
\hline & & 41975 & 141907 & P. crassifolia & I. nitidus & M5 & Xianmi & MH121663 & MH124466 & MH124528 \\
\hline & & 41978 & & P. crassifolia & I. nitidus & M5 & Xianmi & MH121664 & MH124467 & MH124529 \\
\hline & & 41979 & 141908 & P. crassifolia & I. nitidus & M5 & Xianmi & MH121665 & $\mathrm{MH} 124468$ & - \\
\hline \multirow[t]{6}{*}{8} & O. bicolor & 41861 & & P. crassifolia & I. nitidus & - & Maixiu & MH121666 & MH124469 & - \\
\hline & & 41877 & & P. crassifolia & 1. nitidus & M15 & Maixiu & MH121667 & $\mathrm{MH} 124470$ & $\mathrm{MH} 124530$ \\
\hline & & 41878 & & P. crassifolia & P. poligraphus & M20 & Maixiu & MH121668 & $\mathrm{MH} 124471$ & $\mathrm{MH} 124531$ \\
\hline & & 41949 & & P. crassifolia & 1. nitidus & M18 & Xianmi & MH121669 & MH124472 & MH124532 \\
\hline & & 41957 & & P. crassifolia & 1. nitidus & M18 & Xianmi & MH121670 & $\mathrm{MH} 124473$ & $\mathrm{MH} 124533$ \\
\hline & & 41965 & & P. crassifolia & 1. nitidus & M20 & Xianmi & MH121671 & $\mathrm{MH} 124474$ & $\mathrm{MH} 124534$ \\
\hline 9 & $\begin{array}{l}\text { Leptographium } \\
\text { ningerense }\end{array}$ & 41868 & & P. crassifolia & 1. nitidus & M6 & Maixiu & MH121672 & $\mathrm{MH} 124475$ & MH124535 \\
\hline 10 & L. breviuscapum & 41887 & & P. crassifolia & P. poligraphus & M6 & Maixiu & MH121673 & MH124476 & MH124536 \\
\hline \multirow[t]{3}{*}{11} & L. taigense & 41864 & & P. crassifolia & 1. nitidus & - & Xianmi & MH121674 & MH124477 & $\mathrm{MH} 124537$ \\
\hline & & 41970 & & P. crassifolia & I. nitidus & M2 & Xianmi & MH121675 & MH124478 & $\mathrm{MH} 124538$ \\
\hline & & 41977 & & P. crassifolia & 1. nitidus & M5 & Xianmi & MH121676 & MH124479 & MH124539 \\
\hline \multirow[t]{2}{*}{12} & Grosmannia purpurea & 41928 & & P. purpurea & 1. shangrila & M18 & Maixiu & MH121677 & $\mathrm{MH} 124480$ & $\mathrm{MH} 124540$ \\
\hline & & 41922 & & P. purpurea & 1. shangrila & M18 & Maixiu & MH121678 & MH124481 & $\mathrm{MH} 124541$ \\
\hline \multirow[t]{6}{*}{13} & G. zekuensis & 41862 & & P. crassifolia & I. nitidus & - & Maixiu & MH121679 & MH124482 & $\mathrm{MH} 124542$ \\
\hline & & 41870 & 141900 & P. crassifolia & 1. nitidus & M2 & Maixiu & MH121680 & - & MH124543 \\
\hline & & 41871 & & P. crassifolia & 1. nitidus & M6 & Maixiu & MH121681 & MH124483 & MH124544 \\
\hline & & 41875 & & P. crassifolia & 1. nitidus & M9 & Maixiu & MH121682 & MH124484 & MH124545 \\
\hline & & 41876 & $141901^{\mathrm{H}}$ & P. crassifolia & I. nitidus & M16 & Maixiu & MH121683 & MH124485 & $\mathrm{MH} 124546$ \\
\hline & & 48852 & 141902 & P. crassifolia & I. nitidus & M6 & Maixiu & MH121684 & MH124486 & MH124547 \\
\hline \multirow[t]{3}{*}{14} & G. xianmiense & 41866 & & P. crassifolia & 1. nitidus & M13 & Maixiu & MH121685 & MH124487 & MH124548 \\
\hline & & 41867 & & P. crassifolia & 1. nitidus & M18 & Maixiu & MH121686 & MH124488 & MH124549 \\
\hline & & 41880 & & P. crassifolia & P. poligraphus & M20 & Maixiu & MH121687 & MH124489 & $\mathrm{MH} 124550$ \\
\hline
\end{tabular}

${ }^{a}$ The culture collection (CBS) of Westerdijk Fungal Biodiversity Institute, Utrecht, the Netherlands; CMW Culture Collection of the Forestry and Agricultural Biotechnology Institute (FABI), University of Pretoria, Pretoria, South Africa

${ }^{\mathrm{b}}$ Hex-holotype isolate

c Mite species, see Table 1

${ }^{d}$ ITS Internal transcribed spacer regions 1 and 2 of the nuclear ribosomal DNA operon, including the 5.8S region, ITS2-LSU The internal transcribed spacer 2 region and partial large subunit of the nrDNA operon, BT Beta-tubulin, EF Translation elongation factor 1-alpha

The number of fungal isolates collected from different mite species differed substantially (Table S3). About $20 \%$ of the fungi were isolated from mites in the family Winterschmidtiidae. Twenty-four isolates collected from Uropodoidea sp. 4 represented $17 \%$ of the total isolates. This was followed by $12.1 \%$ of the isolates collected from Insectolaelaps sp. 2, 10\% isolates collected from Insectolaelaps sp. 1, 10.6\% isolates collected from Uropodoidea sp. 6 (Table S3).

The 33 isolates collected from the mites in D. micans galleries represented $23.4 \%$ of the total number of isolates, while 75 isolates from the mites in I. nitidus galleries represented 53.2\%, 19 isolates from the mites in $I$. shangrila galleries represented $14.2 \%$, and the remaining 13 isolates from mites in $P$. poligraphus galleries represented $9.2 \%$ of the isolates.

\section{TAXONOMY}

Based on the phylogenetic analyses of different gene regions, as discussed above, three out of the total 14 taxa identified in this study represented novel species, and descriptions for these novel species are provided below. Phylogenetic analyses of $B T$ and $E F$ gene regions (Fig. S1) also indicated that $O$. ainoae and $O$. poligraphi belonged to the same species, thus Ophiostoma poligraphi is synonymized with Ophiostoma ainoae. 


\section{Taxon 4}

Ophiostoma kunlunense R.L. Chang \& Z.W. de Beer, sp. nov.

\section{MycoBank MB 827335}

(Fig. 6)

Etymology. Name refers to the Kunlun mountains, which is one of the longest mountain ranges in Asia, that ends in Qinghai province from where this fungus was first isolated.

Diagnosis: Ophiostoma kunlunense is phylogenetically distinct from all other species in the O. piceae complex, and groups closest to species slightly peripheral to the complex like O. floccosum, O. setosum and O. nikkoense. It produces a pesotum-like asexual morph similar to other species in the complex, and species peripheral to the complex such as O. nikkoense and O. setosum. However, both O. kunlunense and O. nikkoense lack the sporothrix-like synasexual state that characterizes O. floccosum, O. setosum and most other species in the complex. In addition, $O$. floccosum is distinguished by yellow conidial masses, while O. kunlunense and all the other species produce white conidial masses (Harrington et al. 2001). Ophiostoma nikkoense is distinguished from O. kunlunense and the other species by its extremely elongated, clavate and septate conidia (Yamaoka et al. 2004).

Type: China: Qinghai province: Maixiu Forest Farm, from Uropodoidea sp. in gallery of Ips shangrila on Picea purpurea, 8 Aug. 2010, S. J. Taerum (PREM61583 holotype (dried culture); CMW41927 = CBS141903 - extype culture).

Description: Sexual morph not observed. Asexual morph pesotum-like, occurring singly or in groups of up to 15 , macronematous, synnematous, erect, (511-) 705-1729.5 (-1301) $\mu \mathrm{m}$ long, including condiogenous apparatus. Conidia hyaline, 1-celled, smooth, oblong, clavate or obovoid (3.5-) 3-6 (-8) × 1.5-2(-2.5) $\mu \mathrm{m}$, accumulating in a white, gelatinous mass at the apex of the synnema.

Culture characteristics: Colonies hyaline or dark brown when synnemata form. Mycelium superficial on the agar. Pesotum-like asexual morph dominant in the cultures. Optimal temperature for growth $20^{\circ} \mathrm{C}$, reaching $56.6 \mathrm{~mm}$ diam in $10 \mathrm{~d}$. No growth observed at $5^{\circ} \mathrm{C}$ or $30^{\circ} \mathrm{C}$ and above.

Additional specimens examined: China: Qinghai province: Maixiu Forest Farm, from Insectolaelaps sp. in gallery of Ips shangrila on Picea purpurea, 8 Aug. 2010, S. J. Taerum (PREM61584-dried culture; CMW48853=CBS141904 - culture); ibid., 8 Aug. 2010, S. J. Taerum(PREM61585 - dried culture; CMW48854 = CBS141905 - culture).

\section{Taxon 5}

Ophiostoma ainoae H. Solheim, Nord. J. Bot. 6: 201 (1986).

Synonym: Ophiostoma poligraphi M.L. Yin et al. Fungal Biol. 120: 464 (2016).

Notes: When $B T$ and $E F$ sequences produced for several isolates obtained in the present study were analysed together with sequences of three $O$. ainoae isolates from the study of Linnakoski et al. (2016a) and two O. poligraphi isolates from the study of Yin et al. (2016), it became clear that the latter two groups of isolates represented geographically isolated populations of the same species. Sequences of our isolates did not group consistently with isolates in either of the two clades (Fig. $\mathrm{S} 1$ ). For the $B T$ region, our isolates grouped between the two 'species', while in the EF analyses, they all grouped with $O$. poligraphi. There are confirmed reports of $O$. ainoae from Ips typographus and Pityogenes chalcographus on Picea abies in Europe (Linnakoski et al. 2016a), while O. poligraphi was described from Polygraphus poligraphus and Dendroctonus micans on Pi. crassifolia in Qinghai (Yin et al. 2016). Our isolates were from mites on P. poligraphus and Ips nitidus, also from Pi. crassifolia in Qinghai, and this is consistent with the host and beetle vectors of both species. Although a sexual state has not been described for O. poligraphi, the broad synnemata of this species also correspond with those described for O. ainoae (Solheim 1986), supporting the synonymy of the two species.

\section{Taxon 7}

Ophiostoma manchongi R.L. Chang \& Z.W. de Beer, sp. nov.

MycoBank MB 827336

(Fig. 7)

Etymology. Name refers to manchong, the Chinese word for mite.

Diagnosis: Both ITS and BT sequences clearly distinguish between $O$. manchongi and its closest relative, $O$. japonicum. In addition, the sporothrix-like asexual morph of O. manchongi, producing obovoid conidia, is very different from the light coloured synnematous asexual state of O. japonicum with its cylindrical or clavate conidia (Yamaoka et al. 1997). 

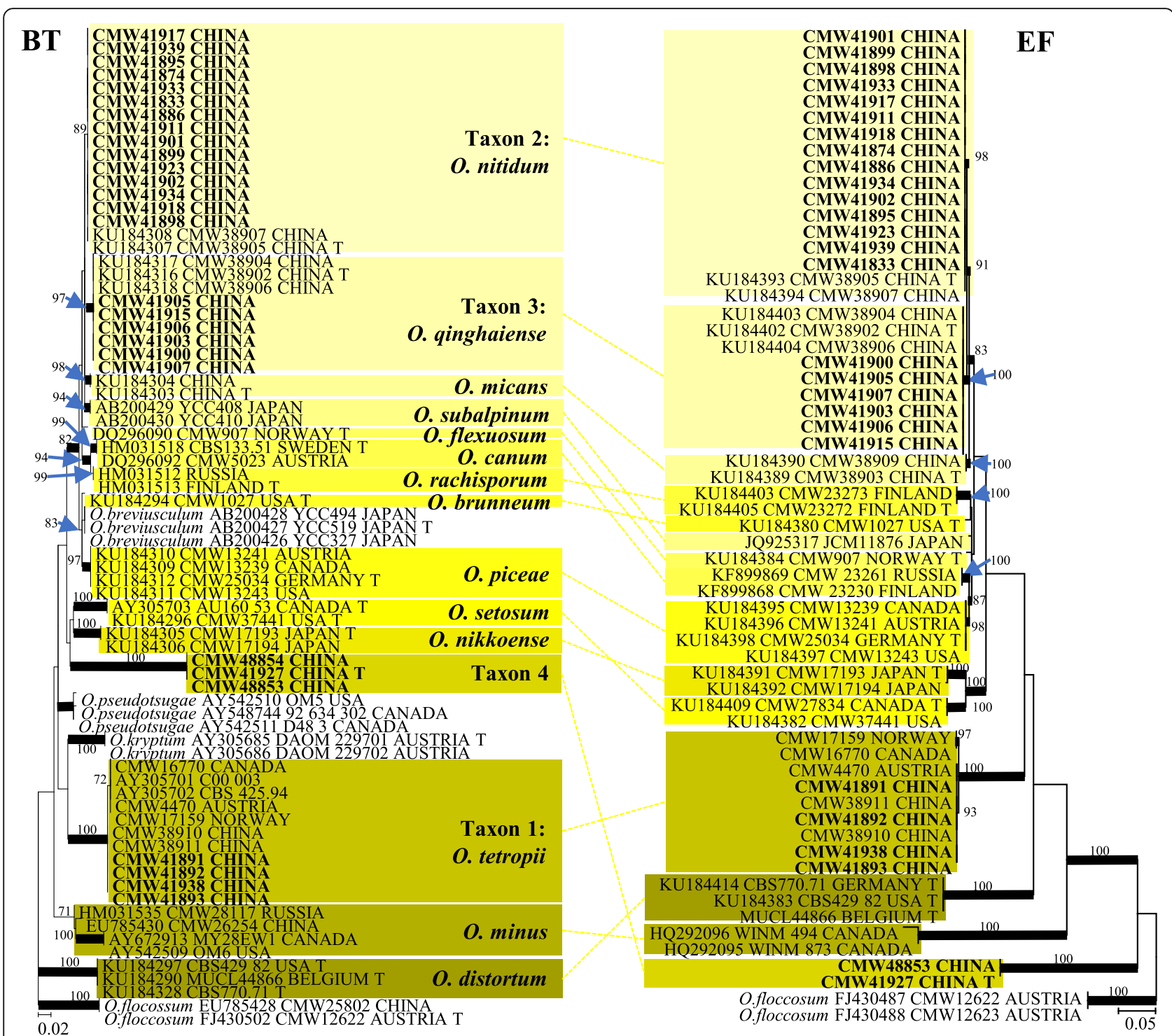

Fig. 3 Phylogram obtained from ML analyses of the partial BT and EF gene of O. piceae complex. Sequences obtained in this study are printed in bold type. ML bootstrap support values (1000 replicates, normal type) above $75 \%$ are indicated at the nodes. Posterior probabilities (above 0.9 ) obtained from $\mathrm{Bl}$ are indicated by bold lines at the relevant branching points. $\mathrm{T}=$ ex-type cultures. Scale bar = total nucleotide difference

between taxa

Type: China: Qinghai province: Xianmi Forest Farm, from Uropodoidea sp. in gallery of Ips shangrila on Picea purpurea, 8 Aug. 2010, S. J. Taerum (PREM61580 holotype (dried culture); CMW41954 = CBS141906 - extype culture).

Description: Sexual morph not observed. Asexual morph sporothrix-like, erect, arising directly from the mycelium. Conidia hyaline, 1-celled, smooth, oblong, obovoid (3-) 4-5.5 (-7) x (1.5-) 2-3 (-4.5) $\mu \mathrm{m}$.

Culture characteristics: colonies at first hyaline, later becoming dark brown at the centre. Mycelium superficial on the agar. Optimal temperature for growth $25^{\circ} \mathrm{C}$, reaching $29.6 \mathrm{~mm}$ diam in $10 \mathrm{~d}$. No growth at $5^{\circ} \mathrm{C}$ or $35^{\circ} \mathrm{C}$.

Specimens examined: China: Qinghai province: Xianmi Forest Farm, from Insectolaelaps sp. in gallery of Ips nitidus on Picea crassifolia, 8 Aug. 2010, S. J. Taerum (PREM61581-dried culture; CMW42975 = CBS141907 culture); ibid., from Uropodoidea sp. in gallery of Ips shangrila on Picea purpurea, 8 Aug. 2010, S. J. Taerum (PREM61582 - dried culture; CMW41979 = CBS141908 - culture).

\section{Taxon 13}

Grosmannia zekuensis R.L. Chang \& Z.W. de Beer, sp. nov. 


\begin{tabular}{|c|c|}
\hline 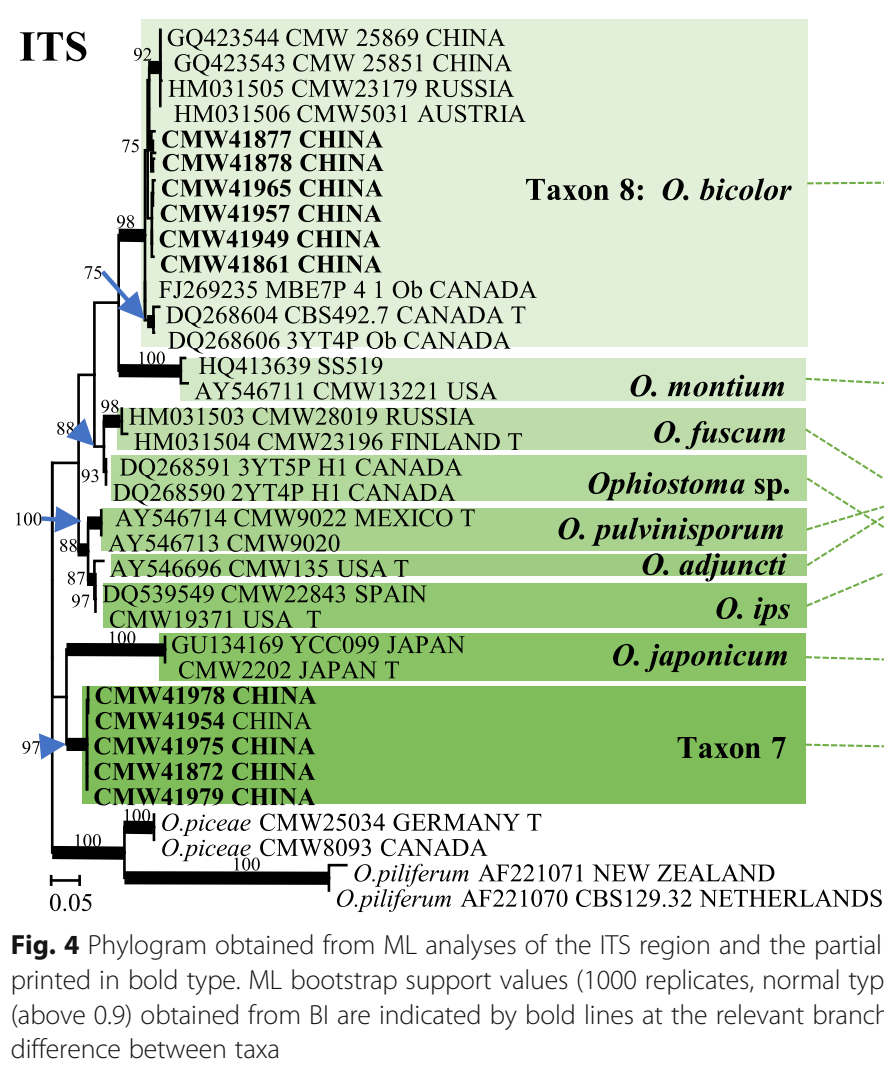 & 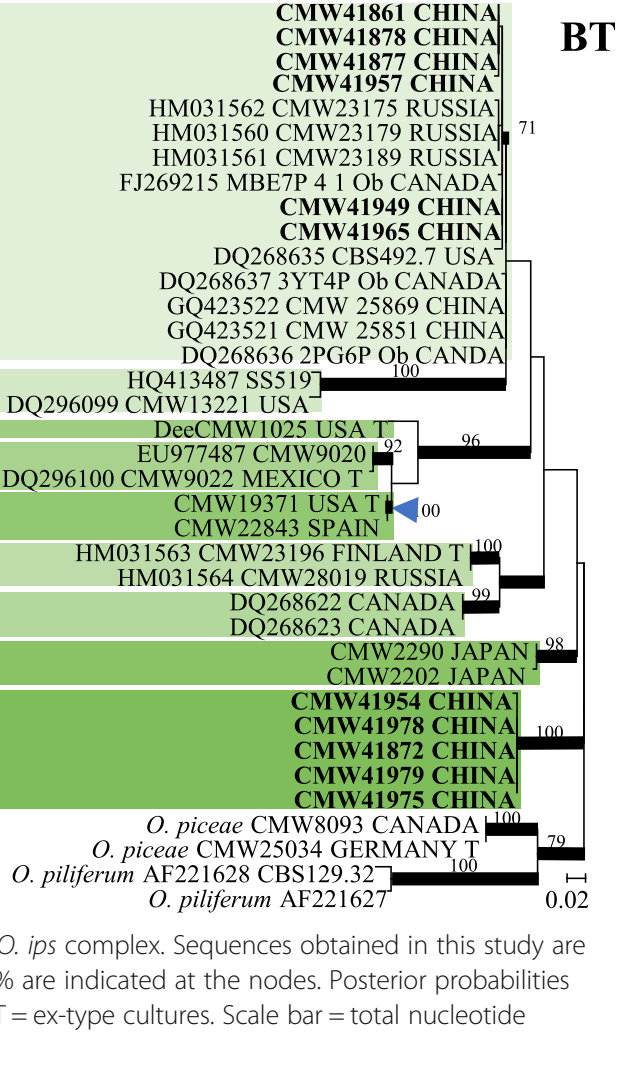 \\
\hline
\end{tabular}

MycoBank MB 827337

(Fig. 8)

Etymology: After Zeku, the county where samples were collected.

Diagnosis: Grosmannia zekuensis is closely related to G. purpurea based on $B T$ and EF sequences, but the two species can be readily distinguished based on morphology and growth rate. G. zekuensis forms smaller asexual structures and conidia than G. purpurea, which is evident when the lengths of the following structures are compared: stipes $67-269$ vs $100-170 \mu \mathrm{m}$; conidiogenous apparatus $16-67$ vs 177-162 $\mu \mathrm{m}$; conidiogenous cells 6-15 vs $70-110 \mu \mathrm{m}$; conidia $3.5-8$ vs $10-15 \mu \mathrm{m}$. However, G. zekuensis grows between 46 and $73 \mathrm{~mm}$ diam on $2 \% \mathrm{MEA}$ in $8 \mathrm{~d}$ at $25^{\circ} \mathrm{C}$ (varying between different isolates), while $G$. purpurea isolates grow on average around $32 \mathrm{~mm}$ diam under the same conditions (Yin et al. 2020).

Type: China: Qinghai province: Xianmi Forest Farm, from Bakerdania sp. in gallery of Ips nitidus on Picea crassifolia, 8 Aug. 2010, S. J. Taerum (PREM61579 holotype (dried culture); CMW41876 = CBS141901 - extype culture).
Description: Sexual morph not observed. Conidiophores macronematous, mononematous, erect, arising directly from the mycelium, (98-) 114-182 (-269) $\mu \mathrm{m}$ long. Rhizoids present. Stipes olivaceous, 2-4 septate, not constricted at septa, (67-) 73.5-125.5 (-182) $\mu \mathrm{m}$ long; apical cells occasionally swollen at apex, (4-) 5-7.5 (10.5) $\mu \mathrm{m}$ wide; basal cells not swollen, (5.5-) 7-10 (11) $\mu \mathrm{m}$ wide. Conidiogenous apparatus (16-) 30.5-49 (-67) $\mu \mathrm{m}$ long, excluding the conidial mass, with multiple series of cylindrical branches; primary branches olivaceous, smooth, cylindrical, not swollen at apex, aseptate, arrangement of primary branches was Type $\mathrm{B}$-more than two branches, (7.5-) 10-16 (-16) x (3-) 3.5-4.5 (-5.5) $\mu \mathrm{m}$; secondary branches light olivaceous, frequently swollen at apex, aseptate, (6-) 8-11 (-12.5) x (2-) 2.5-4 (-5) $\mu \mathrm{m}$; tertiary branches light olivaceous, aseptate, (6-) 7-10 (-11) x (2-) 2.5-3 (-3) $\mu \mathrm{m}$. Conidiogenous cells discrete, hyaline, $2-3$ per branch, aseptate, cylindrical, tapering slightly at the apex, (6-) 8-12 $(-14.5) \times(1.5-) 2-3(-3) \mu \mathrm{m}$. Conidia hyaline, aseptate, elliptical, (3.5-) 4-5.5 (-8) x (2-) 2.5-3 (-3.5) $\mu \mathrm{m}$.

Culture characteristics: Colonies hyaline without asexual structures or dark brown when asexual structures form. Mycelium superficial on the agar. Optimal temperature for growth $25^{\circ} \mathrm{C}$. Some isolates grow faster, reaching 


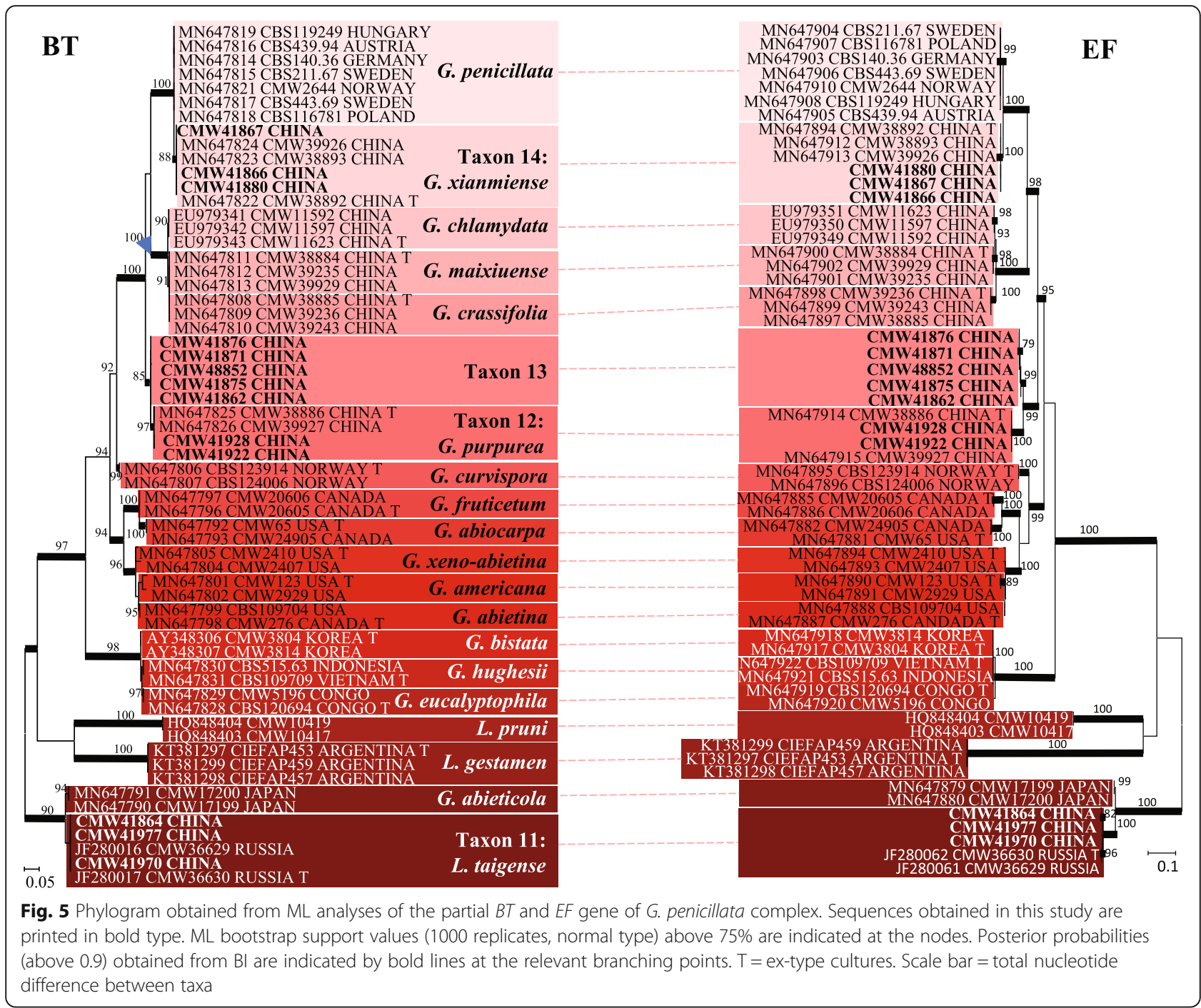

$73.3 \mathrm{~mm}$ diam in $8 \mathrm{~d}$ at $25^{\circ} \mathrm{C}$ and no growth at $30^{\circ} \mathrm{C}$ and above. However, some isolates grow slowly and only reach $46.3 \mathrm{~mm}$ diam in $8 \mathrm{~d}$ and slow growth observed at $35^{\circ} \mathrm{C}$.

Additional specimens examined: China: Qinghai province: Xianmi Forest Farm, from Insectolaelaps sp. in gallery of Ips nitidus on Picea crassifolia, 8 Aug. 2010, S. J. Taerum (PREM61578 - dried culture; CMW48852 = CBS141902 - culture); ibid., from Schwiebea wainsteini in gallery of Ips nitidus on Picea crassifolia, 8 Aug. 2010, S. J. Taerum (PREM61577 - dried culture; CMW41870 = CBS141900 - culture).

\section{DISCUSSION}

We collected 173 mites representing 18 species from spruce-infesting bark beetles in Qinghai province, China. Uropodoidea sp. 4 and Uropodoidea sp. 6, were most abundant among the mites. We obtained 135 fungal isolates from 65 mite individuals. Based on DNA sequence data, 14 fungal species were identified, eight species of Ophiostoma and six of Leptographium s. lat. Among these species, three were recognized as novel taxa and were thus described and provided with names. Of the remaining 11 species, ten had previously been reported from China. Ophiostoma tetropii is reported for the first time from China.

The only previous study to report on ophiostomatoid fungi associated with phoretic mites in China was conducted on mite associates of six pine-infesting bark beetles in Yunnan (Chang et al. 2017). The species diversity of mites collected in the present study from Qinghai was higher than that found in Yunnan (18 species vs. 13), despite the fact that only four beetle species were sampled in Qinghai as opposed to six in Yunnan. However, more mite individuals (173) were collected in Qinghai from two host trees than the 106 mites from only Pinus kesiya in Yunnan (Chang et al. 2017). Only 

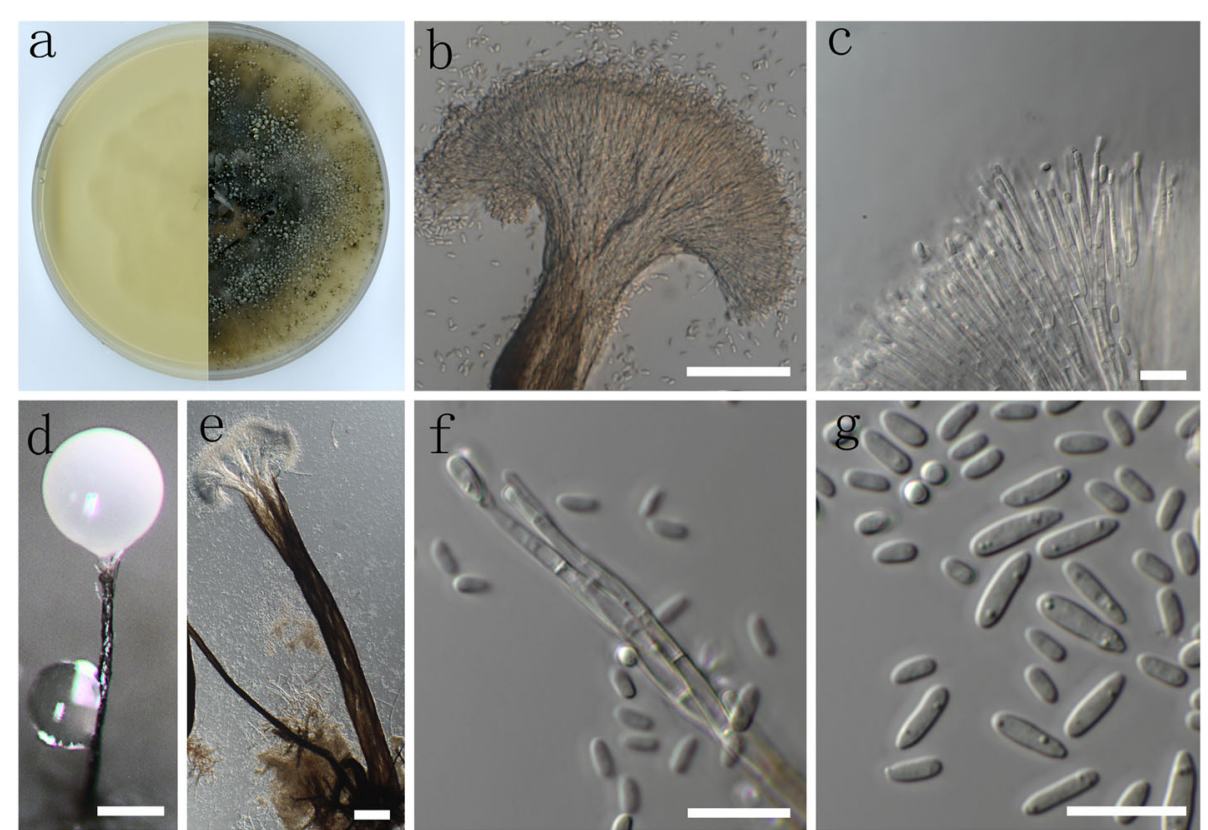

Fig. 6 Morphological characters of asexual structure of Ophiostoma kunlunense sp. nov. a Cultures on malt extract agar (MEA); b, d, e Pesotum asexual state; $\mathbf{c}, \mathbf{f}$ conidiogenous cells; $\mathbf{g}$ conidia. - Scale bars: $\mathbf{b}=50 \mu \mathrm{m} ; \mathbf{c}, \mathbf{f}, \mathbf{g}=10 \mu \mathrm{m} ; \mathbf{d}=200 \mu \mathrm{m} ; \mathbf{e}=100 \mu \mathrm{m}$

Insectolaelaps sp. 1 was collected from both Qinghai and Yunnan. This species was the most frequently collected species in Yunnan and the fourth most frequently collected species in Qinghai, suggesting that it is widely distributed on conifer hosts in western China. The fact that only one species was shared between the two regions is perhaps not surprising because the mites were collected from different bark beetle species, different tree species, and different climatic zones.

Together with the previous report from Yunnan (Chang et al. 2017), a total of 31 species of mites associated with ten bark beetles have now been reported from western China.
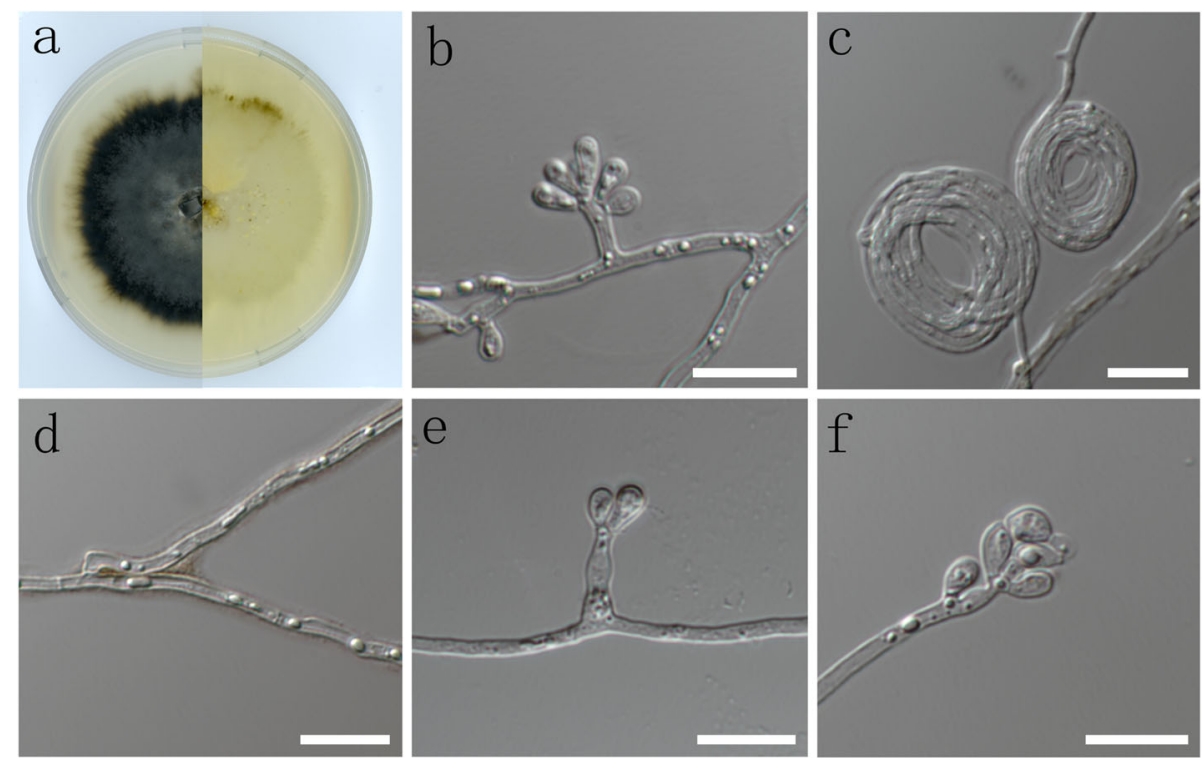

Fig. 7 Morphological characters of asexual structure of Ophiostoma manchongi sp. nov. a Cultures on malt extract agar (MEA); b, e, f conidiogenous cells and conidia; c, d mycelium. -Scale bars: $10 \mu \mathrm{m}$ 

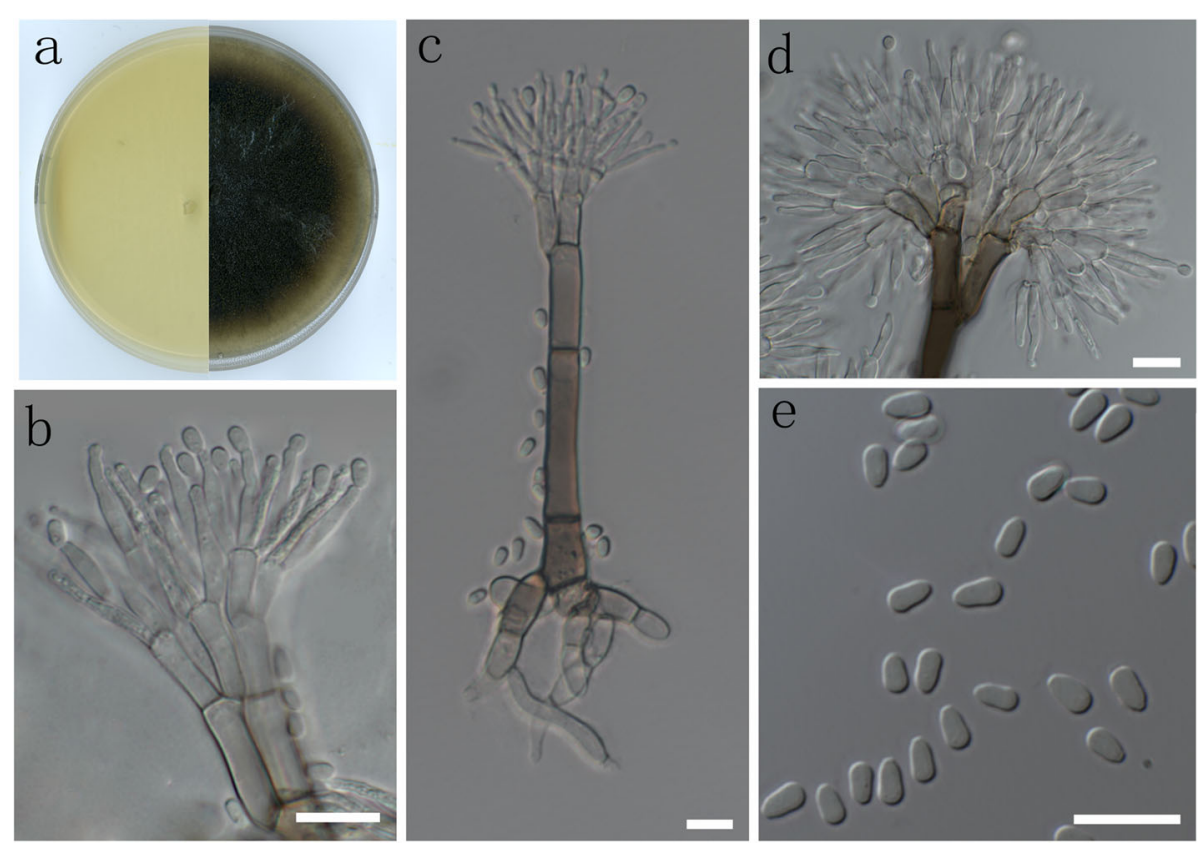

Fig. 8 Morphological characters of asexual structure of Grosmannia zekuensis sp. nov. a Cultures on malt extract agar (MEA); b, d conidiogenous apparatus; c conidiophore; e conidia. Scale bars: $10 \mu \mathrm{m}$

This number is much less than 270 mite species that have been reported in association with more than 110 bark beetle species from the many studies in North America and Europe (Hofstetter et al. 2015), 12 mite species were reported from Ips typographus in Japan, of which only three species did not occur in Europe (Moser et al. 1997). Because most of the mites in our study have not been identified to species level, and because our sampling strategy was aimed at isolating fungi and not only the collection of mites, it is not possible to compare the presence or absence of mite species with other studies. However, at a higher taxonomic level, the most frequent mite order associated with bark beetles was the Mesostigmata in both China and collections collectively from Europe and North America, where it is represented by 20 and 140 species respectively (Hofstetter et al. 2015).

The fungal species diversity from mites in Qinghai was somewhat higher when compared with the diversity of fungal species from mites in Yunnan (Chang et al. 2017), with 12 species reported from Yunnan versus 14 species in Qinghai, although the total number of fungal isolates was smaller in Qinghai. The fungi collected in Qinghai all belonged to only two genera in the Ophiostomatales, Ophiostoma and Leptographium s. lat., while isolates from Yunnan belonged to five genera, four (Graphilbum, Leptographium s. lat., Ophiostoma and Sporothrix) in the Ophiostomatales, and Graphium in the Microascales. The fungal community collected from bark beetle-associate mites in Qinghai was very different to that in Yunnan. Only one fungal species, L. ningerensis, was shared between Qinghai and Yunnan.
A comparison of the fungal species from spruce infesting bark beetles in Qinghai (Yin et al. 2016) with those from mites in our study, revealed that eight species were found in both studies. These included: L. breviuscapum, G. purpurea, G. xianmiense, O. nitidum, O. qinghaiense, $O$. ainoae, O. shangrilae and O. tetropii. Not surprisingly, a large number of fungal species were also shared between galleries and mites in Yunnan province (Chang et al. 2017).

Apart from the three new species discovered in this study, we also collected and identified $O$. tetropii for the first time from China. This fungus was first described from spruce tree attacked by Tetropium sp. in Sweden (Mathiesen 1951), and was subsequently also reported from Picea abies attacked by Ips typographus and Pityogenes chalcographus in Finland (Linnakoski et al. 2010) and Picea rubens attacked by Tetropium fuscum in Canada (Harrison and Smith 2013). Its presence on mites in China is surprising because it was considered as an associate and indicator of beetles such as T. fuscum (Harrison and Smith 2013).

\section{CONCLUSIONS}

The results of this study conducted in China reaffirmed the findings elsewhere in the world showing that there are close associations between bark beetle-associated mites and ophiostomatoid fungi. The sampling area, beetle species and hosts considered was relatively limited. Yet many undescribed species of mites and various novel taxa in Ophiostoma and Leptographium emerged from the study. This reflects how little is 
known regarding the ecology of bark beetles and their symbionts. This is not only in China but also in many other parts of the world. Although relatively high numbers of mites and fungal species were found in in this study, the survey covered a relatively small geographical area. Larger surveys with more systematic sampling are needed in the future to elucidate the ecological roles and specificity of fungus-vector relationships. However, what is clear is that mites may act as important vectors of fungal species other than those vectored by the bark beetles. These mites and their fungi should therefore also be considered in pest risk assessments which often only focus on the bark beetles and their fungi.

\section{Supplementary information}

Supplementary information accompanies this paper at https://doi.org/10. 1186/s43008-020-00037-9

Additional file 1: Fig. S1. Phylogram obtained from ML analyses of the partial BT and EF gene of Ophiostoma clavatum complex. Sequences obtained in this study are printed in bold type. ML and MP bootstrap support values (1000 replicates, normal type) above $75 \%$ are indicated at the nodes. Posterior probabilities (above 0.9) obtained from BI are indicated by bold lines at the relevant branching points. $T=$ ex-type cultures. Scale bar = total nucleotide difference between taxa.

Additional file 2: Fig. S2. Phylogram obtained from ML analyses of the partial BT and EF gene of Leptographium olivaceum complex. Sequences obtained in this study are printed in bold type. ML bootstrap support values (1000 replicates, normal type) above $75 \%$ are indicated at the nodes. Posterior probabilities (above 0.9) obtained from Bl are indicated by bold lines at the relevant branching points. $T=$ ex-type cultures. Scale bar $=$ total nucleotide difference between taxa.

Additional file 3: Table S1. Ophiostomatoid fungi reported from China. Additional file 4: Table S2. Numbers of mite individuals collected and numbers of mite individuals carring fungi in this study.

Additional file 5: Table S3. Numbers of fungal isolates associated with mites from different beetle galleries. Shades of red indicate number of fungal isolates.

\section{Abbreviations}

BI: Bayesian inference; BT: $\beta$-tubulin; EF: Elongation factor 1-a; FABI: Forestry and Agricultural Biotechnology Institute; ITS: The internal transcribed spacer regions 1 and 2; LSU: The nuclear large subunit; MEA: Malt extract agar; ML: Maximum likelihood; s. lat: sensu lato; s. str.: sensu stricto

\section{Adherence to national and international regulations}

All material for this study was collected in China in 2010, thus before the implementation of the Nagaoya Protocol to the Convention on Biological Diversity.

\section{Authors' contributions}

$\mathrm{RC}$ collected and processed material in the laboratory, analysed data, and wrote the first draft of the manuscript. TAD assisted with data analyses and editing the manuscript. ST collected material and edited the manuscript. MJW assisted in interpretation of results and editing the manuscript. XD facilitated and participated in field collections and editing. ZWDB assisted with data analyses, interpretation of results and writing the manuscript. All authors read and approved the final manuscript.

\section{Funding}

We thank members of Tree Protection and Cooperation Programme (TPCP), the Department of Science and Technology (DST)-National Research Foundations (NRF), Center of Excellence in Tree Health Biotechnology (CTHB), and University of Pretoria, Pretoria, South Africa, for financial support.

\section{Availability of data and materials}

All data generated or analysed during this study are included in this published article [and its supplementary information files].

Ethics approval and consent to participate

Not applicable.

\section{Consent for publication}

Not applicable.

\section{Competing interests}

The authors declare that they have no competing interests.

\section{Author details}

${ }^{1}$ Department of Biochemistry, Genetics \& Microbiology, Forestry and Agricultural Biotechnology Institute (FABI), University of Pretoria, Pretoria 0002, South Africa. ${ }^{2}$ College of Life Sciences, Shandong Normal University, Jinan 250014, China.

Received: 29 May 2020 Accepted: 23 June 2020

Published online: 30 July 2020

\section{References}

Ayres MP, Wilkens RT, Ruel JJ, Lombardero MJ, Vallery E (2000) Nitrogen budgets of phloem-feeding bark beetles with and without symbiotic fungi. Ecology 81:2198-2210

Barras SJ (1970) Antagonism between Dendroctonus frontalis and the fungus Ceratocystis minor. Annals of the Entomological Society of America 63:11871190

Bateman C, Huang Y-T, Simmons DR, Kasson MT, Stanley EL, Hulcr J (2017) Ambrosia beetle Premnobius cavipennis (Scolytinae: Ipini) carries highly divergent ascomycotan ambrosia fungus, Afroraffaelea ambrosiae gen. nov. et sp. nov. (Ophiostomatales). Fungal Ecology 25:41-49

Brasier C (1990) China and the origins of Dutch elm disease: an appraisal. Plant Pathology 39:5-16

Brasier CM (1991) Ophiostoma novo-ulmi sp. nov., causative agent of current Dutch elm disease pandemics. Mycopathologia 115:151-161

Bridges JR (1983) Mycangial fungi of Dendroctonus frontalis (Coleoptera, Scolytidae) and their relationship to beetle population trends. Environmental Entomology 12:858-861

Chang R, Duong TA, Taerum SJ, Wingfield MJ, Zhou X, de Beer ZW (2017) Ophiostomatoid fungi associated with conifer-infesting beetles and their phoretic mites in Yunnan, China. MycoKeys 28:19-64

Chang R, Duong TA, Taerum SJ, Wingfield MJ, Zhou XD, De Beer ZW (2019) Ophiostomatoid fungi associated with the spruce bark beetle Ips typographus, including 11 new species from China. Persoonia 42:50-74

Darriba D, Taboada GL, Doallo R, Posada D (2012) jModelTest 2: more models, new heuristics and parallel computing. Nature Methods 9:772-772

De Beer ZW, Wingfield MJ (2013) Emerging lineages in the Ophiostomatales. In: Seifert KA, De Beer ZW, Wingfield MJ (eds) The ophiostomatoid fungi: expanding frontiers CBS, Utrecht, pp 21-46.

De Beer ZW, Duong TA, Wingfield MJ (2016a) The divorce of Sporothrix and Ophiostoma: solution to a problematic relationship. Studies in Mycology 83: 165-191

De Beer ZW, Marincowitz S, Duong TA, Kim J-J, Rodrigues A, Wingfield MJ (2016b) Hawksworthiomyces gen. nov. (Ophiostomatales), illustrates the urgency for a decision on how to name novel taxa known only from environmental nucleic acid sequences (ENAS). Fungal Biology 120:1323-1340

De Beer ZW, Seifert KA, Wingfield MJ (2013) The ophiostomatoid fungi: their dual position in the Sordariomycetes. In: Seifert KA, De Beer ZW, Wingfield MJ (eds) The ophiostomatoid fungi: expanding frontiers. CBS, Utrecht, pp 1-19

Duong TA, De Beer ZW, Wingfield BD, Wingfield MJ (2012) Phylogeny and taxonomy of species in the Grosmannia serpens complex. Mycologia 104: $715-732$

Gardes M, Bruns TD (1993) ITS primers with enhanced specificity for basidiomycetes-application to the identification of mycorrhizae and rusts. Molecular Ecology 2:113-118

Glass NL, Donaldson GC (1995) Development of primer sets designed for use with the PCR to amplify conserved genes from filamentous Ascomycetes. Applied and Environmental Microbiology 61:1323-1330 
Goldhammer DS, Stephen FM, Paine TD (1990) The effect of the fungi Ceratocystis minor (Hedgecock) Hunt var. barrasii Taylor, and SJB 122 on reproduction of the southern pine beetle, Dendroctonus frontalis Zimmermann (Coleoptera: Scolytidae). Canadian Entomologist 122:407-418

Harrington TC, McNew D, Steimel J, Hofstra D, Farrell R (2001) Phylogeny and taxonomy of the Ophiostoma piceae complex and the Dutch elm disease fungi. Mycologia 93:111-136

Harrison K, Smith G (2013) The discovery of Ophiostoma tetropii with the brown spruce longhorn beetle (Tetropium fuscum) in Halifax, Canada. In: Seifert KA, De Beer ZW, Wingfield MJ (eds) The ophiostomatoid fungi: expanding frontiers. CBS, Utrecht, pp 213-217

Hofstetter RW, Dinkins-Bookwalter J, Davis TS, Klepzig KD (2015) Symbiotic associations of bark beetles. In: Vega FE, Hofstetter RW (eds) Bark beetles: biology and ecology of native and invasive species. Academic, San Diego, pp 209-245

Hofstetter RW, Klepzig KD, Moser JC, Ayres MP (2006) Seasonal dynamics of mites and fungi and their interaction with southern pine beetle. Environmental Entomology 35:22-30

Hofstetter RW, Moser JC (2014) The role of mites in insect-fungus associations. Annual Review of Entomology 59:537-557

Hofstetter RW, Moser JC, Blomquist S (2013) Mites associated with bark beetles and their hyperphoretic ophiostomatoid fungi. In: Seifert KA, De Beer ZW, Wingfield MJ (eds) The ophiostomatoid fungi: expanding frontiers. CBS Biodiversity Series, Utrecht, pp 165-176

Jacobs K, Bergdahl DR, Wingfield MJ, Halik S, Seifert KA, Bright DE, Wingfield BD (2004) Leptographium wingfieldii introduced into North America and found associated with exotic Tomicus piniperda and native bark beetles. Mycological Research 108:411-418

Katoh K, Standley DM (2013) MAFFT multiple sequence alignment software version 7: improvements in performance and usability. Molecular Biology and Evolution 30:772-780

Kirisits T (2004) Fungal associates of european bark beetles with special emphasis on the ophiostomatoid fungi. In: Lieutier F, Day K, Battisti A, Grégoire JC, Evans $\mathrm{H}$ (eds) Bark and wood boring insects in living trees in Europe, a synthesis. Kluwer Academic Publishers, Dordrecht, pp 181-236

Klepzig KD, Hofstetter RW (2011) From attack to emergence: interactions between southern pine beetle, mites, microbes, and trees. In: Coulson RN, Klepzig KD (eds) Southern pine beetle II. Department of Agriculture Forest Service, United States, pp 141-152

Levieux J, Lieutier F, Moser JC, Perry TJ (1989) Transportation of phytopathogenic fungi by the bark beetle Ips sexdentatus Boerner and associated mites. Journal of Applied Entomology 108:1-11

Li Z, Zou J, Mao K, Lin K, Li H, Liu J, Källman T, Lascoux M (2012) Population genetic evidence for complex evolutionary histories of four high altitude juniper species in the Qinghai-Tibetan Plateau. Evolution 66:831-845

Linnakoski R, De Beer ZW, Ahtiainen J, Sidorov E, Niemela P, Pappinen A, Wingfield MJ (2010) Ophiostoma spp. associated with pine- and spruceinfesting bark beetles in Finland and Russia. Persoonia 25:72-93

Linnakoski R, De Beer ZW, Niemelä P, Wingfield MJ (2012) Associations of coniferinfesting bark beetles and fungi in Fennoscandia. Insects 3:200-227

Linnakoski R, Jankowiak R, Villari C, Kirisits T, Solheim H, de Beer ZW, Wingfield MJ (2016a) The Ophiostoma clavatum species complex: a newly defined group in the Ophiostomatales including three novel taxa. Antonie Van Leeuwenhoek 109:987-1018

Linnakoski R, Mahilainen S, Harrington A, Vanhanen H, Eriksson M, Mehtätalo L, Pappinen A, Wingfield MJ (2016b) Seasonal succession of fungi associated with Ips typographus beetles and their phoretic mites in an outbreak region of Finland. PLoS One 11:e0155622

Liu L, Wu J, Luo Y, Li Z, Wang G, Han F (2008) Morphological and biological investigation of two pioneer Ips bark beetles in natural spruce forests in Qinghai Province, northwest China. Forestry Studies in China 10:19-22

Liu XW, Wang HM, Lu Q, Decock C, Li YX, Zhang XY (2017) Taxonomy and pathogenicity of Leptographium species associated with Ips subelongatus infestations of Larix spp. in northern China, including two new species. Mycological Progress 16:1-13

Lombardero MJ, Klepzig KD, Moser JC, Ayres MP (2000) Biology, demography and community interactions of Tarsonemus (Acarina: Tarsonemidae) mites phoretic on Dendroctonus frontalis (Coleoptera: Scolytidae). Agricultural and Forest Entomology 2:193-202

Lu M, Zhou XD, De Beer ZW, Wingfield MJ, Sun JH (2009a) Ophiostomatoid fungi associated with the invasive pine-infesting bark beetle, Dendroctonus valens, in China. Fungal Diversity 38:133-145
Lu Q, Decock C, Zhang XY, Maraite H (2009b) Ophiostomatoid fungi (Ascomycota) associated with Pinus tabuliformis infested by Dendroctonus valens (Coleoptera) in northern China and an assessment of their pathogenicity on mature trees. Antonie Van Leeuwenhoek 96:275-293

Marincowitz S, Duong TA, De Beer ZW, Wingfield MJ (2015) Cornuvesica: a little known mycophilic genus with a unique biology and unexpected new species. Fungal Biology 119:615-630

Mathiesen A (1951) Einige neue Ophiostoma-Arten in Schweden. Svensk Botanisk Tidskrift 45:203-232

Miller MA, Pfeiffer W, Schwartz T (2010) Creating the CIPRES science gateway for inference of large phylogenetic trees. In: 2010 Gateway computing environments workshop (GCE). Institute of Electrical and Electronics Engineers, New Orleans, pp 1-8

Moser JC (1985) Use of sporothecae by phoretic Tarsonemus mites to transport ascospores of coniferous bluestain fungi. Transactions of the British Mycological Society 84:750-753

Moser JC, Eidmann HH, Regnander JR (1989a) The mites associated with Ips typographus in Sweden. Annales Entomologici Fennici 55:23-27

Moser JC, Konrad H, Blomquist SR, Kirisits T (2010) Do mites phoretic on elm bark beetles contribute to the transmission of Dutch elm disease? Naturwissenschaften 97:219-227

Moser JC, Konrad H, Kirisits T, Carta LK (2005) Phoretic mites and nematode associates of Scolytus multistriatus and Scolytus pygmaeus (Coleoptera : Scolytidae) in Austria. Agricultural and Forest Entomology 7:169-177

Moser JC, Perry TJ, Furuta K (1997) Phoretic mites and their hyperphoretic fungi associated with flying Ips typographus japonicus Niijima (Col., Scolytidae) in Japan. Journal of Applied Entomology 121:425-428

Moser JC, Perry TJ, Solheim H (1989b) Ascospores hyperphoretic on mites associated with Ips typographus. Mycological Research 93:513-517

Paciura D, De Beer ZW, Jacobs K, Zhou XD, Ye H, Wingfield MJ (2010a) Eight new Leptographium species associated with tree-infesting bark beetles in China. Persoonia 25:94-108

Paciura D, Zhou XD, De Beer ZW, Jacobs K, Ye H, Wingfield MJ (2010b) Characterisation of synnematous bark beetle-associated fungi from China, including Graphium carbonarium sp. nov. Fungal Diversity 40:75-88

Roets F, Wingfield MJ, Crous PW, Dreyer LL (2007) Discovery of fungus-mite mutualism in a unique niche. Environmental Entomology 36:1226-1237

Roets F, Wingfield MJ, Crous PW, Dreyer LL (2009) Fungal radiation in the Cape Floristic Region: an analysis based on Gondwanamyces and Ophiostoma. Molecular Phylogenetetics and Evolution 51:111-119

Roets F, Wingfield MJ, Wingfield BD, Dreyer LL (2011) Mites are the most common vectors of the fungus Gondwanamyces proteae in Protea infructescences. Fungal Biology 115:343-350

Ronquist F, Teslenko M, van der Mark P, Ayres DL, Darling A, Höhna S, Larget B, Liu L, Suchard MA, Huelsenbeck JP (2012) MrBayes 3.2: efficient Bayesian phylogenetic inference and model choice across a large model space. Systematic Biology 61:539-542

Six DL (2012) Ecological and evolutionary determinants of bark beetle-fungus symbioses. Insects 3:339-366

Solheim H (1986) Species of Ophiostomataceae isolated from Picea abies infested by the bark beetle Ips typographus. Nordic Journal of Botany 6:199-207

Solheim H (1991) Oxygen deficiency and spruce resin inhibition of growth of blue stain fungi associated with Ips typographus. Mycological Research 95:1387-1392

Stamatakis A (2014) RAxML version 8: a tool for phylogenetic analysis and postanalysis of large phylogenies. Bioinformatics 30:1312-1313

Swofford DL (2002) PAUP*. Phylogenetic analysis using parsimony (*and other methods). 4.0b10. Sinauer Associates, Sunderland

Taerum SJ, Duong TA, De Beer ZW, Gillette N, Sun JH, Owen DR, Wingfield MJ (2013) Large shift in symbiont assemblage in the invasive red turpentine beetle. PLOS One 8:e78126

Van der Linde JA, Six DL, De Beer ZW, Wingfield MJ, Roux J (2016) Novel ophiostomatalean fungi from galleries of Cyrtogenius africus (Scolytinae) infesting dying Euphorbia ingens. Antonie Van Leeuwenhoek 109:589-601

Vilgalys R, Hester M (1990) Rapid genetic identification and mapping of enzymatically amplified ribosomal DNA from several Cryptococcus species. Journal of Bacteriology 172:4238-4246

Wang H, Lu Q, Meng X, Liu X, Decock C, Zhang X (2016) Ophiostoma olgensis, a new species associated with Larix spp. and Ips subelongatus in northern China. Phytotaxa 282:282-290

Wang H, Lun Y, Lu Q, Liu H, Decock C, Zhang X (2018) Ophiostomatoid fungi associated with pines infected by Bursaphelenchus xylophilus and Monochamus alternatus in China, including three new species. MycoKeys 39:1-27 
Wang H, Wang Z, Liu F, Wu CX, Zhang SF, Kong Xiang B, Decock C, Lu Q, Zhang Z (2019) Differential patterns of ophiostomatoid fungal communities associated with three sympatric Tomicus species infesting pines in southwestern China, with a description of four new species. MycoKeys 50:93-133

Wang Z, Liu Y, Wang H, Meng X, Liu X, Decock C, Zhang X, Lu Q (2020) Ophiostomatoid fungi associated with Ips subelongatus, including eight new species from northeastern China. IMA Fungus 11:3

Webber JF (2004) Experimental studies on factors influencing the transmission of Dutch elm disease. Forest Systems 13:197-205

Wegensteiner R, Wermelinger B, Herrmann M (2015) Natural enemies of bark beetles: predators, parasitoids, pathogens, and nematodes. In: Vega FE, Hofstetter RW (eds) Bark beetles: biology and ecology of native and invasive species. Academic, San Diego, pp 247-304

White TJ, Bruns T, Lee S, Taylor JW (1990) Amplification and direct sequencing of fungal ribosomal RNA genes for phylogenetics. In: Innis MA, Gelfand DH, Sninsky JJ, White TJ (eds) PCR protocols: a guide to methods and applications. Academic, San Diego, pp 315-322

Yamaoka Y, Masuya H, Ohtaka N, Kaneko S, Abe J-iP (2004) Three new Ophiostoma species with Pesotum anamorphs associated with bark beetles infesting Abies species in Nikko, Japan. Mycoscience 45:277-286

Yamaoka Y, Wingfield MJ, Takahashi I, Solheim H (1997) Ophiostomatoid fungi associated with the spruce bark beetle Ips typographus f. japonicus in Japan. Mycological Research 101:1215-1227

Yin M, Wingfield MJ, Zhou X, De Beer ZW (2020) Phylogenetic re-evaluation of the Grosmannia penicillata complex (Ascomycota, Ophiostomatales), with the description of five new species from China and USA. Fungal Biology 124 110-124

Yin M, Wingfield MJ, Zhou X, Linnakoski R, De Beer ZW (2019) Taxonomy and phylogeny of the Leptographium olivaceum complex (Ophiostomatales, Ascomycota), including descriptions of six new species from China and Europe. MycoKeys 60:93-123

Yin ML, Duong TA, Wingfield MJ, Zhou XD, De Beer ZW (2015) Taxonomy and phylogeny of the Leptographium procerum complex, including Leptographium sinense sp. nov. and Leptographium longiconidiophorum sp. nov. Antonie Van Leeuwenhoek 107:1-17

Yin ML, Wingfield MJ, Zhou XD, De Beer ZW (2016) Multigene phylogenies and morphological characterization of five new Ophiostoma spp. associated with spruce-infesting bark beetles in China. Fungal Biology 120:454-470

Zhou X, Song R, Zhou X, Cui L, Cao C (2011) Fungal population in the inside and outside of Ips subelongatus body and the gallary of insect-bored larch logs. Mycosystema 30:400-407

Zhou XD, De Beer ZW, Wingfield MJ (2013) Ophiostomatoid fungi associated with conifer-infecting bark beetles in China. In: Seifert KA, De Beer ZW, Wingfield MJ (eds) Ophiostomatoid fungi: expanding frontiers. CBS, Utrecht, pp 91-98

\section{Publisher's Note}

Springer Nature remains neutral with regard to jurisdictional claims in published maps and institutional affiliations.

Ready to submit your research? Choose BMC and benefit from:

- fast, convenient online submission

- thorough peer review by experienced researchers in your field

- rapid publication on acceptance

- support for research data, including large and complex data types

- gold Open Access which fosters wider collaboration and increased citations

- maximum visibility for your research: over $100 \mathrm{M}$ website views per year

At $\mathrm{BMC}$, research is always in progress.

Learn more biomedcentral.com/submissions 